\title{
Classical ground states of symmetric Heisenberg spin systems
}

\author{
Heinz-Jürgen Schmidt调 and Marshall Luban \\ † Universität Osnabrück, Fachbereich Physik, Barbarastr. 7, 49069 Osnabrück, \\ Germany \\ $\ddagger$ Ames Laboratory and Department of Physics and Astronomy, Iowa State University \\ Ames, Iowa 50011, USA
}

\begin{abstract}
We investigate the ground states of classical Heisenberg spin systems which have point group symmetry. Examples are the regular polygons (spin rings) and the seven quasi-regular polyhedra including the five Platonic solids. For these examples, ground states with special properties, e. g. coplanarity or symmetry, can be completely enumerated using group-theoretical methods. For systems having coplanar (anti-) ground states with vanishing total spin we also calculate the smallest and largest energies of all states having a given total spin $S$. We find that these extremal energies depend quadratically on $\mathrm{S}$ and prove that, under certain assumptions, this happens only for systems with coplanar $S=0$ ground states. For general systems the corresponding parabolas represent lower and upper bounds for the energy values. This provides strong support and clarifies the conditions for the so-called rotational band structure hypothesis which has been numerically established for many quantum spin systems.
\end{abstract}

$\S$ To whom correspondence should be addressed (hschmidt@uos.de) 


\section{Introduction}

The study of small interacting spin systems is not only of theoretical interest but also of importance for the experimental investigation of recently synthetized magnetic molecules [1], [2], [3], [4]. An exact calculation of the thermal expectation values of the relevant quantum observables and other quantities such as correlation functions is possible only in very few cases. Given this situation, a classical treatment yields a first approximation for individual spins $s$ with $s \gg 1$ which is often astonishingly good, as well as bounds for the exact quantum values (c. f. Berezin/Lieb-inequalities). It is also valuable as a guide for developing approximation schemes for attacking the quantum theoretical problem.

One of the fundamental characteristics of a system is its ground state(s) and the corresponding ground state energy. The problem of determining the exact classical ground state has been considered for a long time, see e. g. [5], [6], but has only been solved for a few special systems. These include arrays of interacting spins occupying the sites of a regular $N$-polygon (spin rings) with nearest-neighbor coupling constant $J>0$ (antiferromagnetic in our convention). The corresponding ground state energy is

$$
E_{\text {min }}=-2 J N s^{2},(\text { even } N),
$$

corresponding to an alternating configuration of spin orientations in the $N$-polygon. The analogous result

$$
E_{\text {min }}=2 J N s^{2} \cos ((N-1) \pi / N),(\operatorname{odd} N)
$$

does not appear to be in the literature, although it is easily established, see (73). Another class of systems where the classical ground states are easily determined are the so-called bi-partite systems, which can be divided into two subsets $A$ and $B$ such that there are only interactions (with a positive coupling constant $J$ ) between spins $a \in A$ and $b \in B$. In this case any anti-parallel configuration between $A$ and $B$ will minimize the energy. The regular polygons with even $N$ considered above and the cube are simple examples of bi-partite systems.

Recently, the exact classical ground state of a system consisting of $N=30$ spins occupying the vertices of an icosidodecahedron [7], [8] has been determined [9]. The method employed there can be applied to any system which can be decomposed into a set of triangles without common edges and which is 3-colorable. Examples are the octahedron (as mentioned in [9]) and the cuboctahedron (this is obtained by joining the mid-points of the edges of a cube with their nearest neighbours, see figure 6). All these systems have coplanar ground states with $S=0$ which can be modified to remain ground states in the presence of a magnetic field $\mathcal{H}$, the corresponding energy depending quadratically on $\mathcal{H}$, c. f. [9]. This yields a definite prediction for the magnetisation versus magnetic field plot at low temperatures: It should have a constant slope until a critical magnetic field $\mathcal{H}_{c}$ is applied, and for $\mathcal{H}>\mathcal{H}_{c}$ the magnetisation is saturated. 
In the present paper we will derive a further consequence for systems having coplanar ground states with $S=0$. The same formal equation as that characterizing the ground state in the presence of a magnetic field occurs for the ground state subject to the extra constraint that the square of the total spin assumes a given value. Generalizing the solution in [9] we obtain a whole family of states assuming the minimal energy $E_{\min }(S)$. It turns out that for these systems the function $E_{\text {min }}(S)$ is quadratic in $S$ of the form

$$
E_{\text {min }}(S)=\frac{j-j_{\min }}{N} S^{2}+j_{\min } N
$$

Here $j$ is the row sum $\left(j=\sum_{\nu} J_{\mu \nu}\right)$ of the adjacency matrix $\mathbb{J}$ defined below. $\mathbb{J}$ is chosen in such a way that $j$ does not depend on $\mu$, and $j_{\min }$ is the smallest eigenvalue of $\mathbb{J}$. Conversely, the existence of a lower RBS-parabola (3) implies the coplanarity of the ground state, see theorem 2 for details. Analogous results hold for the maximal eigenvalue $E_{\text {max }}(S)$ of systems with $S=0$ coplanar anti-ground states. Hence these systems show an exact rotational band structure (RBS) which has been conjectured to apply for a general class of quantum spin systems [10]. Thus our findings strongly support that conjecture in the sense that the considered class of spin systems has exact RBS-parabolas in the classical limit $s \rightarrow \infty$. On the other hand we have found classes of systems which deviate from the exact RBS-parabolas, especially for small values of $S$, see section 6 . Hence our work may be viewed as a first step towards an understanding of the conditions for the occurence of RBS-parabolas. We will call systems, which exactly attain their lower RBS-parabola (3), "parabolic". For general systems the corresponding parabolas represent lower and upper bounds for the energy values, as will be proved in section 4. Sometimes these bounding parabolas will be very good approximations of the boundaries of the energy spectrum even for non-parabolic systems.

Other questions arise in connection with spin systems having coplanar $S=0$ ground states. Has every classical spin system coplanar ground states or even coplanar $S=0$ ground states? If not, what is the criterion for having coplanar ground states? Are there systems with both coplanar and non-coplanar ground states? We do not have complete answers to these questions, but we can show the following:

- In general the spin triangle with arbitrary coupling constants $J_{1}, J_{2}, J_{3}$ has only coplanar ground states with $S>0$.

- A spin system which we call "pentagonal star" has a non-coplanar ground state with $S>0$. The existence of coplanar states with the same energy can be excluded on the basis of numerical calculations.

- For certain systems the existence of non-coplanar, symmetric ground states can be excluded. By "symmetric" we mean roughly that the state has the same symmetry as the spin system itself (for details see section 5).

- Other systems, such as the dodecahedron and the icosahedron, have symmetric non-coplanar ground states and apparently no coplanar ones. These ground states 
can be geometrically vizualized in terms of what is known [7] as the "great stellated dodecahedron" and the "great icosahedron", respectively.

- The tetrahedron has a symmetric non-coplanar ground state with $S=0$ and a variety of other non-symmetric ground states including coplanar ones, all of the same energy.

- The same is true for other systems with full permutational symmetry (" $N$ pantahedron"), except that these systems do not have symmetric ground states for $N>4$.

- The cuboctahedron has a lower RBS-parabola but interestingly it has two symmetric ground states with $S=0$, a coplanar one and a non-coplanar one. The latter corresponds to a degenerate stellated figure which we have not found in the literature and which is composed of four Stars of David, see figure 8 .

Thus we are faced with a surprising variety of possibilities of ground states of different types. The main open problem is to rigorously prove the existence of spin systems without coplanar ground states. It appears that quantitative statements can be made mainly if symmetric states are concerned, since in this case we may use standard methods of group theory, in particular representation theory of the point groups.

The article is organized as follows: Section 2 contains the general definitions and results which are independent of symmetry assumptions. The main result is that systems with coplanar $S=0$ ground states have RBS-parabolas (theorem 1) and its converse (theorem 2). These parabolas represent lower and upper energy bounds even for nonparabolic systems, as proven in section 4 . In section 3 we address questions of extension of ground states to larger systems and "higher order frustration". Section 5 introduces symmetry assumptions and the definition and simple properties of symmetric states. This machinery is applied in section 6 to the investigation of particular examples. We consider the general triangle which is generically non-parabolic as well as classes of symmetric spin systems including the quasi-regular polyhedra. We construct symmetric ground states from certain irreducible representations of the respective point groups. The article closes with a summary.

\section{Notations, definitions, and general results}

We consider as the phase space of the classical spin system the $N$-fold product of the unit sphere $\mathcal{S}^{2}$. Instead of using canonical coordinates we will denote a state by a sequence $\boldsymbol{s}$ of unit vectors $\boldsymbol{s}_{\mu}, \mu=1, \ldots, N$ with components $s_{\mu}^{i}, i=1,2,3$. The total spin is $\boldsymbol{S} \equiv \sum_{\mu} \boldsymbol{s}_{\mu}$ with components $\boldsymbol{S}^{i}, i=1,2,3$. If not indicated otherwise, the abstract letters $i, j, \ldots$ will always be (upper) indices and there is no danger of confusion with the square of a vector, e. g. $\boldsymbol{S}^{2}$. We will use bracketed indices to denote by, say, $x^{(i)}$ the vector with the components $x^{i}$, similarly for matrices. For example, the $n \times m$ matrix $A_{(i)(j)}$ consists of $n$ row vectors $A_{i(j)}$ and of $m$ column vectors $A_{(i) j}$. 
If not mentioned otherwise, the Hamilton function (or "energy") will be of the form

$$
H_{0}(\boldsymbol{s})=\sum_{\mu \nu} J_{\mu \nu} \boldsymbol{s}_{\mu} \cdot \boldsymbol{s}_{\nu}
$$

If a magnetic field $\overrightarrow{\mathcal{H}}$ is to be included we add a Zeeman term and obtain

$$
H_{h}=H_{0}-\boldsymbol{h} \cdot \boldsymbol{S}
$$

where $\boldsymbol{h} \equiv \sqrt{s(s+1)} g \mu_{B} \overrightarrow{\mathcal{H}}$ contains the common combination of the spectroscopic splitting factor $g$ and the Bohr magneton $\mu_{B}$ and is scaled with the function $\sqrt{s(s+1)}$ of the corresponding spin quantum number $s$.

Note that the exchange parameters $J_{\mu \nu}$ are not uniquely determined by the Hamiltonian $H_{0}$ via (4). Different choices of the $J_{\mu \nu}$ leading to the same $H_{0}$ will be referred to as different "gauges". We will adopt the following gauges. First, the antisymmetric part of $\mathbb{J}$ does not occur in the Hamiltonian (4). Hence we will follow common practice and choose $J_{\mu \nu}=J_{\nu \mu}$. Thus the $J_{\mu \nu}$ can be considered as the entries of a real symmetric matrix $\mathbb{J}$. Second, since $\mathbf{s}_{\mu} \cdot \mathbf{s}_{\mu}=1$ we may choose arbitrary diagonal elements $J_{\mu \mu}$ without changing $H_{0}$, as long as their sum vanishes, $\operatorname{Tr} \mathbb{J}=0$. The usual gauge chosen throughout the literature is $J_{\mu \mu}=0, \mu=1, \ldots, N$, which will be called the "zero gauge". In this article, however, we will choose another gauge, to be called the "homogeneous gauge", which is defined by the condition that the row sums

$$
J_{\mu} \equiv \sum_{\nu} J_{\mu \nu}
$$

will be independent of $\mu$. Note that the eigenvalues of $\mathbb{J}$ may non-trivially depend on the gauge. We found that the eigenvalues of $\mathbb{J}$ are only relevant for energy estimates if the row sum of $\mathbb{J}$ is constant. This would restrict the applicability of large parts of our theory if we adopt the zero gauge. However, by introducing the homogeneous gauge we can apply our results to a very general class of systems.

The quantity

$$
N j \equiv \sum_{\mu \nu} J_{\mu \nu}
$$

is gauge-independent. If exchange parameters satisfying $\widetilde{J}_{\mu \nu}=\widetilde{J}_{\nu \mu}$ are given in the zero gauge, the corresponding parameters $J_{\mu \nu}$ in the homogeneous gauge are obtained as follows:

$$
\begin{aligned}
& J_{\mu \nu} \equiv \widetilde{J}_{\mu \nu} \text { for } \mu \neq \nu, \\
& J_{\mu \mu} \equiv j-\widetilde{J}_{\mu} .
\end{aligned}
$$

It follows that

$$
j=J_{\mu}=\sum_{\nu} \widetilde{J}_{\mu \nu}+J_{\mu \mu}
$$


A spin system is called antiferromagnetic (AF) iff all $J_{\mu \nu} \geq 0$ for $\mu \neq \nu$. A system is called connected iff its spin sites cannot be decomposed into two disjoint classes, say $\mathcal{A}$ and $\mathcal{B}$, such that $J_{\mu \nu}=0$ if $\mu \in \mathcal{A}$ and $\nu \in \mathcal{B}$.

In the special case where all exchange parameters $J_{\mu \nu}, \mu \neq \nu$ are 0 or $J$, the system can be essentially represented by its graph $\Gamma$. A graph $\Gamma=(\mathcal{V}, \mathcal{E})$ consists of a set of "vertices" $\mathcal{V}$ and a set of "edges" $\mathcal{E} \subset[\mathcal{V}]^{2}$. Here $[\mathcal{V}]^{2}$ denotes the set of subsets of $\mathcal{V}$ with exactly 2 elements. In our case, the vertices are the spin sites, $\mathcal{V}=\{1, \ldots, N\}$, and the edges represent interacting pairs of spins. For spin systems it is appropriate to consider only graphs without loops and multiple edges, as we do in this article, following, e. g. , 11].

There are different ways to graphically represent a spin state $\boldsymbol{s}_{\mu}$. For specific spin systems, e. g. magnetic molecules, the spin sites $\mu \in \mathcal{V}$ are embedded into the physical 3 -space and represented by vectors $\boldsymbol{r}_{\mu}$. One way would be to attach the vectors $\boldsymbol{s}_{\mu}$ as small arrows to the sites given by $\boldsymbol{r}_{\mu}$. This is done in figure 7 below. However, when using this representation it is difficult to visualize the structure of the $\boldsymbol{s}_{\mu}$ in spin space. Hence we will mostly employ another method of representation: We draw the $N$ vertices in spin space according to the unit vectors $\boldsymbol{s}_{\mu}$ and join them with lines according to the edges of the original spin graph $\Gamma$. Thus we obtain a graph isomorphic to $\Gamma$ which contains the additional information about the considered spin configuration.

In graph theory the set of edges is often represented by a matrix called the adjacency matrix. We will use this name also for the matrix $\mathbb{J}$ in the general case of different exchange parameters. There exists an extended literature about the connection between the structure of a graph and the spectrum of $\mathbb{J}$, see, for example [12], where also applications in chemistry and physics are mentioned.

The graph $\Gamma$ is called complete if $\mathcal{E}=[\mathcal{V}]^{2}$. We will also call the corresponding spin system where any two spins interact with equal strength, a pantahedron.

Being symmetrical, $\mathbb{J}$ has a complete set of $N$ real, ordered eigenvalues $j_{\min }, \ldots, j_{\max }$ which are counted according to their multiplicity. One of them is the row sum $j$ with $\mathbf{1} \equiv \frac{1}{\sqrt{N}}(1,1, \ldots, 1)$ as the corresponding eigenvector. For connected AF-systems, $j$ will be non-degenerate and equals the largest eigenvalue, $j=j_{\max }>0$, by the theorem of Frobenius-Perron. It will be convenient to rearrange the indices such that $j=j_{0}$ and the remaining eigenvalues $j_{1}, \ldots, j_{N-1}$ are ordered according to their size. Note that for AF systems, $j_{1}=j_{\min }$ since $j_{\min }=j>0$ contradicts $\operatorname{Tr} \mathbb{J}=0$. However, since it may happen that $j_{\max }=j$ we will write $j_{\text {maxi }}$ for the largest eigenvalue of $\mathbb{J}$ other than $j$, counted once, hence $j_{N-1}=j_{\text {maxi }}$ for AF systems. Sums over $\alpha=1, \ldots, N-1$ but excluding $\alpha=0$ will be denoted by $\sum^{\prime}$. We will denote the $\alpha$-th normalized eigenvector 
of $\mathbb{J}$ by $C_{\alpha}^{(\nu)}$, i. e.

$$
\sum_{\nu} J_{\mu \nu} C_{\alpha}^{\nu}=j_{\alpha} C_{\alpha}^{\mu}, \quad \mu, \alpha=0, \ldots, N-1
$$

and

$$
\sum_{\mu} \overline{C_{\alpha}^{\mu}} C_{\beta}^{\mu}=\delta_{\alpha \beta}, \quad \alpha, \beta=0, \ldots, N-1
$$

where we also allow for the possibility to choose complex eigenvectors.

We note in passing that $\mathbb{I}$ in the homogeneous gauge is essentially the Hamilton operator of the corresponding quantum spin system, restricted to the subspace of total magnetic quantum number $M=N s-1$. Thus there is an unexpected connection between "weakly symmetric" classical states to be defined below and quantum eigenstates of the Hamiltonian lying in the mentioned subspace.

The equations of motion resulting from the Hamiltonian (4) are

$$
\frac{d}{d t} \boldsymbol{s}_{\mu}=\left(\sum_{\nu} J_{\mu \nu} \boldsymbol{s}_{\nu}\right) \times \boldsymbol{s}_{\mu} .
$$

Using $J_{\mu \nu}=J_{\nu \mu}$ one can immediately show that the total spin vector $\boldsymbol{S}$ is a constant of motion.

We now will define various kinds of special states. A stationary state will be one with $\frac{d}{d t} \boldsymbol{s}_{\mu}=0$, for all $\mu=1, \ldots, N$. According to (13), this is equivalent to

$$
\sum_{\nu} J_{\mu \nu} \boldsymbol{s}_{\nu}=\kappa_{\mu} \boldsymbol{s}_{\mu}
$$

for some real numbers $\kappa_{\mu}, \mu=1, \ldots, N$. Equation (14) can also be viewed as the solution of the problem to seek states with vanishing variation of the quadratic form

$$
\sum_{\mu \nu} J_{\mu \nu} \boldsymbol{s}_{\mu} \cdot \boldsymbol{s}_{\nu}
$$

subject to the constraints $\boldsymbol{s}_{\mu} \cdot \boldsymbol{s}_{\mu}=1, \quad \mu=1, \ldots, N$. The $\kappa_{\mu}$ then appear as Lagrange parameters of this variational problem.

Geometrically, (14) means that for a stationary state each spin vector is proportional to the "center of mass" of its neighbors. As for general Hamiltonian systems, the stationary states are just the critical points of the Hamilton function, i. e. those points where the gradient of $H_{0}$ vanishes. In particular, the states $\boldsymbol{s}$ with minimal $H_{0}(\boldsymbol{s})=E_{\min }$ or maximal $H_{0}(\boldsymbol{s})=E_{\max }$ are stationary. The former will be called ground states, and the latter anti-ground states. For AF systems the anti-ground state is always of the form $\boldsymbol{s}_{\mu}=\boldsymbol{e}$ for all $\mu=1, \ldots, N$, i. e. all spins are aligned parallel to an arbitrary unit vector $\boldsymbol{e}$ (ferromagnetic ordering). In this case $j_{\max }=j$ and $E_{\max }=N j$. 
Moreover, we will consider the relative ground states, (relative anti-ground states), which are defined as the states of minimal (maximal) energy among all states satisfying $\left(\sum_{\nu} s_{\nu}\right)^{2}=S^{2}$. Let $E_{\min }(S)$ denote the energies of the relative ground states, and $E_{\max }(S)$ that of the relative anti-ground states. Again, the relative ground states and anti-ground states are among the solutions of the variational problem corresponding to (15) with the additional constraint $\left(\sum_{\nu} s_{\nu}\right)^{2}=S^{2}>0$. If the extra Lagrange parameter is called $\chi$, we obtain the condition, analogous to (14),

$$
\sum_{\nu} J_{\mu \nu} \boldsymbol{s}_{\nu}=\kappa_{\mu} \boldsymbol{s}_{\mu}+\chi \boldsymbol{S} \quad \text { for } S>0
$$

The equations of motion (13) then imply that for states satisfying (16) we have

$$
\frac{d}{d t} \boldsymbol{s}_{\mu}=\chi \boldsymbol{S} \times \boldsymbol{s}_{\mu}
$$

i. e. , for $S>0$ these states are no longer stationary but are precessing around the total spin vector. This is completely analogous to the precession of stationary states of $H_{0}$ in the presence of a magnetic field.

The condition $S^{2}>0$ in (16) is necessary in order to apply the method of Lagrange parameters. $S=0$ has to be excluded since it would correspond to a critical point of the constraining function $g(\boldsymbol{s})=\left(\sum_{\nu} \boldsymbol{s}_{\nu}\right)^{2}-S^{2}$, see for example [13. To cover also the case $S=0$ we consider three constraining equations written in vector form

$$
\sum_{\nu} \boldsymbol{s}_{\nu}=\boldsymbol{S}_{0}
$$

where $\boldsymbol{S}_{0}$ is a fixed vector. Then the above-mentioned problem does not occur. We have three Lagrange parameters which will be written as the components of a vector $\boldsymbol{L}$. The resulting equations are

$$
\sum_{\nu} J_{\mu \nu} \boldsymbol{s}_{\nu}=\kappa_{\mu} \boldsymbol{s}_{\mu}+\boldsymbol{L}
$$

Taking the vector product of $(19) \times \boldsymbol{s}_{\mu}$ and summing over $\mu$ yields $\mathbf{o}=\boldsymbol{S} \cdot \boldsymbol{L}$. If $\boldsymbol{S} \neq \mathbf{o}$ this is equivalent to $\boldsymbol{L}=\chi \boldsymbol{S}$ and we recover (16). For $S=0$, however, (16) has to be replaced by (19).

States satisfying (19) will be called weakly stationary states, since they are stationary in a rotating frame. Hence relative (anti-) ground states are weakly stationary.

There exists a simple mechanical model for stationary states: Consider a system of $N$ rigid, massless rods of unit length fixed at the same point ("pendula"). These rods may be represented by unit vectors $\boldsymbol{s}_{\mu}$. Between two rods $\boldsymbol{s}_{\mu}$ and $\boldsymbol{s}_{\nu}$ we consider springs satisfying Hooke's law with spring constants $2 J_{\mu \nu}$. Further there is a constant ("gravitational") force $\boldsymbol{F}=\boldsymbol{F} \boldsymbol{e}$. By the cosine theorem, $\left|\boldsymbol{s}_{\mu}-\boldsymbol{s}_{\nu}\right|^{2}=2\left(1-\boldsymbol{s}_{\mu} \cdot \boldsymbol{s}_{\nu}\right)$, hence the potential energy of our mechanical model will be

$$
V(\boldsymbol{s})=\frac{1}{2} \sum_{\mu \nu} J_{\mu \nu}\left|\boldsymbol{s}_{\mu}-\boldsymbol{s}_{\nu}\right|^{2}-\boldsymbol{F} \cdot \boldsymbol{S}=N j-\sum_{\mu \nu} J_{\mu \nu} \boldsymbol{s}_{\mu} \cdot \boldsymbol{s}_{\nu}-\boldsymbol{F} \cdot \boldsymbol{S} .
$$


The equilibrium points of this mechanical system will satisfy the zero-force equation

$$
2 \sum_{\mu \nu} J_{\mu \nu} \boldsymbol{s}_{\nu}+\boldsymbol{F}=2 \kappa_{\mu} \boldsymbol{s}_{\mu}
$$

where $2 \kappa_{\mu} \boldsymbol{s}_{\mu}$ are essentially the constraining forces exerted on the rigid rods. Moreover, for equilibrium $\boldsymbol{S}$ will be proportional to the constant force, say $\boldsymbol{F}=-2 \chi \boldsymbol{S}$, since otherwise there would be a first-order variation of the potential energy when performing infinitesimal uniform rotations of the system. It follows that the equations (21) and (16) are formally identical. Summarizing, there is a 1:1 correspondence between the weakly stationary spin states $s$ and the equilibrium states $s$ of the considered mechanical systems, if the value of the force $F$ runs through all real numbers. This mechanical model may help the reader to vizualize some of the weakly stationary states to be considered in this article.

We can easily obtain the following bounds for the energy of stationary states $s$ :

Lemma $1 N j_{\min } \leq E_{\min } \leq H_{0}(s)=\sum_{\mu} \kappa_{\mu} \leq E_{\max } \leq N j_{\max }$. Moreover, for AF systems and $S=0$ we have $H_{0}(\boldsymbol{s}) \leq N j_{\max i}$.

Proof: $H_{0}(\boldsymbol{s})=\sum_{\mu \nu} J_{\mu \nu} \boldsymbol{s}_{\nu} \cdot \boldsymbol{s}_{\mu}=\sum_{\mu} \kappa_{\mu} \boldsymbol{s}_{\mu} \cdot \boldsymbol{s}_{\mu}=\sum_{\mu} \kappa_{\mu}$. Further we have $\sum_{\mu \nu} J_{\mu \nu} \boldsymbol{s}_{\nu}$. $\boldsymbol{s}_{\mu} \leq j_{\max } \sum_{\mu} \boldsymbol{s}_{\mu} \cdot \boldsymbol{s}_{\mu}=N j_{\max }$, and analogously for the lower bound. Since (anti)ground states are stationary, these bounds also hold for $E_{\min }$ and $E_{\max }$. The upper energy bound for $S=0$ and AF systems follows from $\sum_{\mu \nu} J_{\mu \nu} \boldsymbol{s}_{\nu} \cdot \boldsymbol{s}_{\mu} \leq j_{\operatorname{maxi}} \sum_{\mu} \boldsymbol{s}_{\mu} \cdot \boldsymbol{s}_{\mu}$, since for $\boldsymbol{S}=\mathbf{o}$ the vectors $s_{(\mu)}^{i}, i=1,2,3$ are orthogonal to the eigenvector $\mathbf{1}$ corresponding to the eigenvalue $j$.

The respective bounds of lemma 1 are attained if there exist stationary states where all $\kappa_{\mu}=j_{\min }$ or $\kappa_{\mu}=j_{\max }$, or $\kappa_{\mu}=j_{\operatorname{maxi}}$ in the AF case for $S=0$. At present, we cannot show the existence of such states under general conditions but this can be done for some specific examples. We will call any stationary state with the property that the values of all $\kappa_{\mu}$ of (14) are independent of $\mu$, a weakly symmetric state. If we view

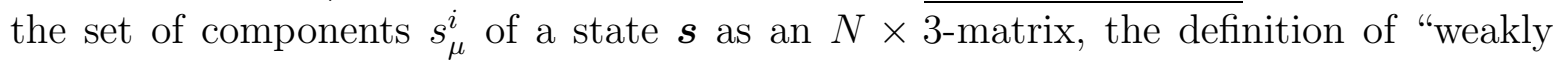
symmetric" could be rephrased in the way that all rows of $s$ are eigenvectors of $\mathbb{J}$ for the same eigenvalue $\widetilde{j}$. Recall that for stationary states each spin vector $\boldsymbol{s}_{\mu}$ is proportional to the "center of mass" of the neighbouring spins. For weakly symmetric states the constant of proportionality will be the same for all spins $\boldsymbol{s}_{\mu}$, which motivates the wording. Obviously, weakly symmetric (anti-) ground states belong to the eigenvalue $j_{\min },\left(j_{\max }\right)$. If one takes any three eigenvectors of $\mathbb{J}$ with the same eigenvalue to form the 3 rows of a matrix, one will not get automatically a weakly symmetric state, since the $N$ columns need not be unit vectors. In section 5 we will present a construction procedure which, under certain assumptions, yields (weakly) symmetric states.

Another simple property of weakly symmetric ground states is the following: 
Lemma 2 Every weakly symmetric state has vanishing total spin if the corresponding eigenvalue $\widetilde{j}$ is different from the row sum $j$. In particular, every weakly symmetric ground state of an AF system has vanishing total spin.

Proof: We obtain $\sum_{\mu \nu} J_{\mu \nu} \boldsymbol{s}_{\nu}=j \sum_{\nu} \boldsymbol{s}_{\nu}=j \boldsymbol{S}$ and $\sum_{\mu \nu} J_{\mu \nu} \boldsymbol{s}_{\nu}=\widetilde{j} \sum_{\mu} \boldsymbol{s}_{\mu}=\widetilde{j} \boldsymbol{S}$. Hence $\widetilde{j}=j$ or $\boldsymbol{S}=\mathbf{o}$. If $\boldsymbol{s}$ is a weakly symmetric ground state of an AF system with $\widetilde{j}=j_{\text {min }}$, we have $j_{\min }<j$ as above.

We will call a state $\boldsymbol{s}$ collinear if all its spin vectors are (anti-)parallel to a given vector $\boldsymbol{e}$, i. e. $\boldsymbol{s}_{\mu}= \pm \boldsymbol{e}$. Due to equation (14), every collinear state is stationary. Further we will call a state $s$ coplanar if all its spin vectors ly in a plane i. e. , if there exists a non-zero 3 -vector $\boldsymbol{n}$ such that $\boldsymbol{n} \cdot \boldsymbol{s}_{\mu}=0$ for all $\mu=1, \ldots, N$. In section 6 we will show that systems such as the regular polygon, the pantahedron and the quasi-regular cuboctahedron and icosidodecahedron admit coplanar weakly symmetric $S=0$ ground states. These ground states are important since one can construct from them a whole family of relative ground states, which show a rotational band structure.

Theorem 1 (i) Let $\boldsymbol{s}$ be a coplanar weakly symmetric state with $S=0$ corresponding to an eigenvalue $\widetilde{j}$ of $\mathbb{J}$ and perpendicular to a unit vector $\boldsymbol{n}$. Then the following family of states $\hat{\boldsymbol{s}}(S), 0 \leq S \leq N$ has total spin length $S$ :

$$
\hat{\boldsymbol{s}}_{\mu}(S)=\sqrt{1-\frac{S^{2}}{N^{2}}} \boldsymbol{s}_{\mu}+\frac{S}{N} \boldsymbol{n}, \quad \mu=1, \ldots, N .
$$

(ii) If, moreover, $\boldsymbol{s}$ is a ground state then $\hat{\boldsymbol{s}}(S)$ will be a relative ground state for all $0 \leq S \leq N$. In this case,

$$
E_{\text {min }}(S)=\frac{j-j_{\text {min }}}{N} S^{2}+j_{\min } N
$$

(iii) The analogous case for $s$ being a coplanar weakly symmetric relative anti-ground state for $S=0$ : Then $\hat{\boldsymbol{s}}(S)$ will be a relative anti-ground state and

$$
E_{\max }(S)=\frac{j-j_{\operatorname{maxi}}}{N} S^{2}+j_{\max i} N
$$

\section{Proof:}

(i) Obviously, $\hat{\boldsymbol{s}}(S)$ is a state with total spin length $S$.

(ii) We obtain $\sum_{\nu} J_{\mu \nu} \hat{\boldsymbol{s}}_{\nu}=\sqrt{1-\frac{S^{2}}{N^{2}}} j_{\min } \boldsymbol{s}_{\mu}+\frac{S}{N} j \boldsymbol{n}$. This is of the form (16) if we choose $\chi=\frac{j-j_{\min }}{N}$. In order to prove that $\hat{\boldsymbol{s}}$ is a relative ground state, we note that $\hat{\boldsymbol{s}}$ is a weakly symmetric ground state w. r. t. the modified adjacency matrix $\dot{J}_{\mu \nu} \equiv J_{\mu \nu}-\chi$, since $j_{\min }$ is also the smallest eigenvalue of $\dot{J}$. The calculation of $E_{\text {min }}(S)$ is straightforward.

(iii) This case is largely analogous, but $j_{\text {min }}$ has to be replaced by $j_{\text {maxi }}$, since $s$ has $S=0$ and cannot be the total anti-ground state. 
Systems which satisfy equation (23) will be called parabolic systems. Hence the essential claim of theorem 1 is that systems with a coplanar $S=0$ ground state will be parabolic.

We will now prove a theorem which can be viewed as the converse of theorem 1 , in so far it shows that coplanar ground states necessarily appear for parabolic systems, if certain technical assumptions are satisfied. We expect that these assumptions hold under fairly general conditions but will not dwell upon this question further.

Theorem 2 Consider a connected AF system and let $t \mapsto \mathbf{s}(t)$ be a smooth curve for $0<t<\epsilon$ of relative ground states such that the limits $t \rightarrow 0$ of $\mathbf{s}(t)$ and its derivatives up to second order exist, in particular $\frac{d S}{d t}(0) \neq 0$ for $S^{2}(t) \equiv\left(\sum_{\mu} \mathbf{s}_{\mu}(t)\right)^{2}$. Moreover, let $\mathbf{s}(0)$ be a weakly symmetric ground state with $S=0$ and assume parabolicity, $E_{\text {min }}(S)=\frac{j-j_{\min }}{N} S^{2}+N j_{\text {min }}$, at least for the interval covered by $0<t<\epsilon$.

Then $\mathbf{s}(0)$ will be coplanar.

Proof: We will indicate the limit $t \rightarrow 0$ of a function of $t$ by skipping the argument, e. g. $\boldsymbol{s}_{\mu} \equiv \boldsymbol{s}_{\mu}(0)$. Differentiation with respect to $t$ will be indicated by an overdot. Since $\boldsymbol{s}_{\mu}(t) \cdot \boldsymbol{s}_{\mu}(t)=1$, differentiation yields

$$
\boldsymbol{s}_{\mu} \cdot \dot{\boldsymbol{s}}_{\mu}=0, \quad \dot{\boldsymbol{s}}_{\mu} \cdot \dot{\boldsymbol{s}}_{\mu}+\boldsymbol{s}_{\mu} \cdot \ddot{\boldsymbol{s}}_{\mu}=0 .
$$

We may assume that $\boldsymbol{S}(t)=S(t) \boldsymbol{e}$ where $\boldsymbol{e}$ is a constant unit vector. Being a weakly stationary relative ground state, $\mathbf{s}(t)$ satisfies (16) for $t>0$ :

$$
\boldsymbol{j}_{\mu}(t) \equiv \sum_{\nu} J_{\mu \nu} \boldsymbol{s}_{\nu}(t)=\kappa_{\mu}(t) \boldsymbol{s}_{\mu}(t)+\chi(t) \sum_{\nu} \boldsymbol{s}_{\nu}(t)
$$

with the limit value $\boldsymbol{S} \equiv \sum_{\nu} \boldsymbol{s}_{\nu}=\mathbf{o}$ according to the assumptions of the theorem. But we cannot a priori assume that $\chi(t)$ has a finite limit value for $t \rightarrow 0$. Thus we solve (26) for $L(t) \equiv S(t) \chi(t)$ and obtain

$$
L(t)=\frac{\boldsymbol{j}_{\mu}(t) \cdot \boldsymbol{e}-\boldsymbol{j}_{\mu}(t) \cdot \boldsymbol{s}_{\mu}(t) \boldsymbol{e} \cdot \boldsymbol{s}_{\mu}(t)}{1-\left(\boldsymbol{e} \cdot \boldsymbol{s}_{\mu}(t)\right)^{2}} .
$$

If $s$ is collinear, the proof is done. If $s$ is not collinear, we find some $\mu$ such that $\left(\boldsymbol{e} \cdot \boldsymbol{s}_{\mu}(t)\right)^{2} \neq 1$ for all $0 \leq t<\epsilon$. For this value of $\mu$, (27) defines a smooth function of $t$, independent of $\mu$, with a finite limit value $L \equiv L(0)$. Moreover, we can solve (26) for $\kappa_{\mu}(t), \mu=1, \ldots, N$ and obtain

$$
\kappa_{\mu}(t)=\sum_{\nu} J_{\mu \nu} \boldsymbol{s}_{\nu}(t) \cdot \boldsymbol{s}_{\mu}(t)-L(t) \boldsymbol{e} \cdot \boldsymbol{s}_{\mu}(t)
$$

This shows that also $\kappa_{\mu}(t)$ is a smooth function of $t$ and has a finite limit for $t \rightarrow 0$. Since, by assumption, $s$ is weakly symmetric this limit must be $\kappa_{\mu}=j_{\text {min }}$ and hence $L=0$. Therefore the smooth function $\chi(t)=\frac{L(t)}{S(t)}$ for $t>0$ has a finite limit $\chi=\lim _{t \rightarrow \infty} \frac{\dot{L}(t)}{\dot{S}(t)}$, employing l'Hospital's rule and that $\dot{S}(0) \neq 0$ by assumption. 
Classical ground states

Differentiating (26) twice and taking the limit $t \rightarrow 0$ yields

$$
\sum_{\nu} J_{\mu \nu} \dot{\boldsymbol{s}}_{\nu}=\dot{\kappa}_{\mu} \boldsymbol{s}_{\mu}+j_{\min } \dot{\boldsymbol{s}}_{\mu}+\chi \dot{\boldsymbol{S}}
$$

and

$$
\sum_{\nu} J_{\mu \nu} \ddot{\boldsymbol{s}}_{\nu}=\ddot{\kappa}_{\mu} \boldsymbol{s}_{\mu}+2 \dot{\kappa}_{\mu} \dot{\boldsymbol{s}}_{\mu}+j_{m i n} \ddot{\boldsymbol{s}}_{\mu}+2 \dot{\chi} \dot{\boldsymbol{S}}+\chi \ddot{\boldsymbol{S}} .
$$

Multipying (26),(29), and (30) with $\boldsymbol{s}_{\mu}$ and summing over $\mu$ yields

$$
\begin{aligned}
H & =\sum_{\mu \nu} J_{\mu \nu} \boldsymbol{s}_{\mu} \cdot \boldsymbol{s}_{\nu}=\sum_{\mu} \kappa_{\mu}+\chi \boldsymbol{S}^{2}=N j_{\text {min }} \\
\frac{1}{2} \dot{H} & =\sum_{\mu \nu} J_{\mu \nu} \dot{\boldsymbol{s}}_{\nu} \cdot \boldsymbol{s}_{\mu}=\sum_{\mu} \dot{\kappa}_{\mu}=\sum_{\nu} j_{\min } \boldsymbol{s}_{\nu} \cdot \dot{\boldsymbol{s}}_{\nu}=0 \\
\frac{1}{2} \ddot{H} & =\sum_{\mu \nu} J_{\mu \nu}\left(\dot{\boldsymbol{s}}_{\nu} \cdot \dot{\boldsymbol{s}}_{\mu}+\boldsymbol{s}_{\mu} \cdot \ddot{\boldsymbol{s}}_{\nu}\right) \\
& =j_{\min } \sum_{\mu} \dot{\boldsymbol{s}}_{\mu}^{2}+\chi \dot{\boldsymbol{S}}^{2}+\sum_{\mu} \ddot{\kappa}_{\mu}+j_{\min } \sum_{\mu} \ddot{\boldsymbol{s}}_{\mu} \cdot \boldsymbol{s}_{\mu} \\
& =\sum_{\mu} \ddot{\kappa}_{\mu}+\chi \dot{\boldsymbol{S}}^{2}
\end{aligned}
$$

using (25) and (29). On the other hand,

$$
\begin{aligned}
& H(t)=\sum_{\mu} \kappa_{\mu}(t)+\chi(t) S^{2}(t), \\
& \dot{H}(t)=\sum_{\mu} \dot{\kappa}_{\mu}(t)+\dot{\chi}(t) S^{2}(t)+2 \chi(t) S(t) \dot{S}(t),
\end{aligned}
$$

and

$$
\ddot{H}=\sum_{\mu} \ddot{\kappa}_{\mu}+2 \chi \dot{S}^{2} .
$$

Comparison with (35) yields $\sum_{\mu} \ddot{\kappa}_{\mu}=0$ and

$$
\ddot{H}=2 \chi \dot{S}^{2} .
$$

Hence, using the assumption of parabolicity and l'Hospital's rule,

$$
\frac{j-j_{\min }}{N}=\left.\frac{d H}{d\left(S^{2}\right)}\right|_{t=0}=\frac{\ddot{H}}{\frac{d^{2}}{d t^{2}} S^{2}}=\frac{2 \chi \dot{S}^{2}}{2 \dot{S}^{2}}=\chi .
$$

Summing (29) over $\mu$ yields

$$
j \dot{\boldsymbol{S}}=\sum_{\mu} \dot{\kappa}_{\mu} \boldsymbol{s}_{\mu}+j_{m i n} \dot{\boldsymbol{S}}+N \chi \dot{\boldsymbol{S}},
$$

whence, by (40) and the assumption $\dot{S} \neq 0$,

$$
\sum_{\mu} \dot{\kappa}_{\mu} \boldsymbol{s}_{\mu}=0 \text {. }
$$


We rewrite (29) in the form

$$
\sum_{\nu}\left(J_{\mu \nu}-j_{\min } \delta_{\mu \nu}-N \chi E_{\mu \nu}\right) \dot{\boldsymbol{s}}_{\nu}=\dot{\kappa}_{\mu} \boldsymbol{s}_{\mu}
$$

where $E$ is the projector onto $\mathbb{J}$ 's eigenvector $\mathbf{1}$ with constant entries $E_{\mu \nu}=\frac{1}{N}$. We expand both sides of (43) into real eigenvectors of $\mathbb{J}$ and obtain

$$
\begin{aligned}
& \dot{\kappa}_{\mu} \boldsymbol{s}_{\mu}=\sum_{\alpha}{ }^{\prime} \boldsymbol{K}_{\alpha} C_{\alpha}^{\mu} \\
& \dot{\boldsymbol{s}}_{\nu}=\sum_{\beta}{ }^{\prime} \frac{1}{j_{\beta}-j} \boldsymbol{K}_{\beta} C_{\beta}^{\mu}+\frac{1}{N} \dot{\boldsymbol{S}} .
\end{aligned}
$$

Now $\sum_{\mu} \dot{\kappa}_{\mu} \boldsymbol{s}_{\mu} \cdot \dot{\boldsymbol{s}}_{\mu}=0, \frac{1}{N} \dot{\boldsymbol{S}} \cdot \sum_{\mu} \dot{\kappa}_{\mu} \boldsymbol{s}_{\mu}=0$, and $\sum_{\mu} C_{\alpha}^{\mu} C_{\beta}^{\mu}=\delta_{\alpha \beta}$ imply

$$
0=\sum_{\mu} \sum_{\alpha}{ }^{\prime} \boldsymbol{K}_{\alpha} C_{\alpha}^{\mu} \cdot \sum_{\beta}{ }^{\prime} \frac{1}{j_{\beta}-j} \boldsymbol{K}_{\beta} C_{\beta}^{\mu}=\sum_{\alpha}{ }^{\prime} \frac{\boldsymbol{K}_{\alpha}^{2}}{j_{\alpha}-j} .
$$

Since $j>j_{\alpha}$ for $\alpha>0$ and connected AF systems, this is only possible if all $\boldsymbol{K}_{\alpha}=\mathbf{o}$. Hence, by (45) $\dot{\boldsymbol{s}}_{\nu}=\frac{1}{N} \dot{\boldsymbol{S}}$ and $\boldsymbol{s}_{\nu} \cdot \frac{1}{N} \dot{\boldsymbol{S}}=0$. Thus $\boldsymbol{s}$ is coplanar.

An analogous theorem concerning $E_{\max }(S)$ and coplanar anti-ground states can be proved similarly.

\section{Extension of ground states}

We consider spin systems with $J_{\mu \nu}=0$ or $J$ and which can thus essentially be characterized by their graph $\Gamma=(\mathcal{V}, \mathcal{E})$. A sub-graph (representing a sub-system) $\widetilde{\Gamma}=(\widetilde{\mathcal{V}}, \widetilde{\mathcal{E}})$ is defined by the conditions $\widetilde{\mathcal{V}} \subset \mathcal{V}, \widetilde{\mathcal{E}} \subset \mathcal{E}$ and $\widetilde{\mathcal{E}} \subset[\widetilde{\mathcal{V}}]^{2}$. Let

$$
\widetilde{H}_{0}=2 J \sum_{(\mu, \nu) \in \widetilde{\mathcal{E}}} \boldsymbol{s}_{\mu} \cdot \boldsymbol{s}_{\nu}
$$

be the Hamiltonian of the subsystem. Let $\boldsymbol{s}$ be any state of $\Gamma$ and $\widetilde{\boldsymbol{s}}$ be its restriction to $\widetilde{\Gamma}$. If $\boldsymbol{s}$ happens to be a ground state of $\Gamma, \widetilde{\boldsymbol{s}}$ need not be a ground state of $\widetilde{\Gamma}$, but, of course, $\widetilde{H}_{0}(\widetilde{\boldsymbol{s}}) \geq \widetilde{E}_{\min }$.

The inverse problem is the extension problem: Given a ground state $\widetilde{\boldsymbol{s}}$ of $\widetilde{\Gamma}$, can it be extended to a ground state $\boldsymbol{s}$ of $\Gamma$ (such that $\widetilde{\boldsymbol{s}}$ is the restriction of $\boldsymbol{s}$ )? In general, this will not be possible. For example, if $\widetilde{\Gamma}$ is the graph of a dimer with ground state $\widetilde{\boldsymbol{s}}=\uparrow \downarrow$, the extension of $\widetilde{\boldsymbol{s}}$ to a global ground state is only possible for bi-partite systems. We have no general theory describing the obstacles against extension (which could be called frustration of higher order), but we can describe the typical situation where extension is possible. For an analogous treatment in the quantum case, see [14].

The situation we have in mind is one where the graph of the spin system $\Gamma_{1}$ is decomposed into smaller graphs which are copies of some graph $\Gamma_{2}$ in such a way that each edge lies in exactly one of the copies. For example, an icosidodecahedron can be 
decomposed into 20 triangles with disjoint edges. We have local states by considering the ground states of the copies of $\Gamma_{2}$. But these local states must fit together in order make it possible to construct a global ground state of $\Gamma_{1}$. For the example of decomposition into triangles the condition of fitting-together of local ground states is equivalent to the 3-colorability of the large system. The icosidodecahedron happens to satisfy this condition and hence possesses a ground state of the energy which is 20 times the ground state energy of the triangle. For general $\Gamma_{2}$ the colorability condition has to be replaced by the existence of a map from $\Gamma_{1}$ to $\Gamma_{2}$ which maps edges onto edges. Such a map may be called a "graph homomorphism".

A graph homomorphism $h: \Gamma_{1} \longrightarrow \Gamma_{2}$ is a pair of maps $h=\left(h_{\mathcal{V}}, h_{\mathcal{E}}\right)$ such that $h_{\mathcal{V}}: \mathcal{V}_{1} \longrightarrow \mathcal{V}_{2}, h_{\mathcal{E}}: \mathcal{E}_{1} \longrightarrow \mathcal{E}_{2}$ and $\{\mu, \nu\} \in \mathcal{E}_{1}$ implies $h_{\mathcal{E}}\{\mu, \nu\}=\left\{h_{\mathcal{V}}(\mu), h_{\mathcal{V}}(\nu)\right\}$. The definition of a graph isomomorphism is analogous.

Proposition 1 Consider two spin systems with graphs $\Gamma_{1}$ and $\Gamma_{2}$ and the following properties

(i) there exists a graph homomorphism $h: \Gamma_{1} \longrightarrow \Gamma_{2}$,

(ii) there exists a decomposition into sub-graphs $\Gamma_{1}=\bigcup_{\mu=1}^{k} \Gamma_{\mu}$, which is a disjoint decomposition with respect to edges,

(iii) the restriction of $h$ to $\Gamma_{\mu}, h_{\mu}: \Gamma_{\mu} \longrightarrow \Gamma_{2}$ is a graph isomorphism for each $\mu=1 \ldots k$.

Moreover, let $\boldsymbol{s}^{(2)}: \mathcal{V}_{2} \longrightarrow \mathcal{S}^{2}$ be a ground state of $\Gamma_{2}$.

Then $\boldsymbol{s}^{(1)} \equiv h_{\mathcal{V}} \circ \boldsymbol{s}^{(2)}$ will be a ground state of $\Gamma_{1}$.

Proof: According to the disjoint decomposition of $\mathcal{E}_{1}, H_{1}$ will be a sum of terms $H_{\mu}$ which are each minimized by the state $\boldsymbol{s}^{(1)}$. Hence $\boldsymbol{s}^{(1)}$ is a ground state of $\Gamma_{1}$.

The above-mentioned construction of ground states for the icosidodecahedron, the octahedron and the cuboctahedron follows the description given in this proposition. Other examples show that the two given conditions of the proposition are essential: The dodecahedron is 3-colorable but it cannot be decomposed into edge-disjoint triangles. Actually its ground state energy is lower than that of the state obtained by the 3coloring. A simpler example is the bi-partite 3-chain which can be mapped by a graph homomorphism onto the triangle but is not isomorphic to the triangle. On the other hand it is possible to connect 4 triangles such that their edges are still disjoint but form a tetrahedron which cannot be 3 -colored. This is then a "higher-order frustrated" system with a ground state energy which is larger than 4 times the ground state energy of a triangle.

\section{Bounding parabolas}

In this section we will show that the parabolas which occur in theorem 1 1 are general bounds for the extremal values $E_{\min }(S)$ and $E_{\max }(S)$, even if the system is not parabolic. 
The proof is completely analogous to the quantum case, see [15]. We recall that $C_{\alpha}^{(\nu)}$ denotes the $\alpha$-th normalized eigenvector of $\mathbb{J}$ and consider a transformation of the spin vectors analogous to the transformation onto the eigenbasis of $\mathbb{J}$. Define

Definition $1 \boldsymbol{T}_{\alpha} \equiv \sum_{\mu} \overline{C_{\alpha}^{\mu}} \boldsymbol{s}_{\mu}$, and $Q_{\alpha} \equiv \overline{\boldsymbol{T}}_{\alpha} \cdot \boldsymbol{T}_{\alpha} \quad \alpha=0, \ldots, N-1$.

The inverse transformation then yields

$$
\boldsymbol{s}_{\mu}=\sum_{\alpha} C_{\alpha}^{\mu} \boldsymbol{T}_{\alpha}, \quad \mu=1, \ldots, N .
$$

Especially, $\boldsymbol{T}_{0}=\boldsymbol{S} / \sqrt{N}$ since $\alpha=0$ corresponds to the eigenvector $\mathbf{1}$. The following lemma follows directly from the definitions:

Lemma $3 N=\sum_{\mu}\left(\boldsymbol{s}_{\mu}\right)^{2}=\sum_{\alpha} Q_{\alpha}=\frac{1}{N} \boldsymbol{S}^{2}+\sum_{\alpha}^{\prime} Q_{\alpha}$.

Our main result of this section is formulated in the following theorem:

Theorem 3 The following inequality holds:

$$
\frac{j-j_{\min }}{N} \boldsymbol{S}^{2}+j_{\min } N \leq H_{0} \leq \frac{j-j_{\operatorname{maxi}}}{N} \boldsymbol{S}^{2}+j_{\max i} N
$$

Proof: We rewrite the Hamilton function in the following form and conclude

$$
\begin{aligned}
H_{0} & =\sum_{\mu \nu \alpha \beta} J_{\mu \nu} \overline{C_{\alpha}^{\mu}} C_{\beta}^{\nu} \overline{\boldsymbol{T}}_{\alpha} \cdot \boldsymbol{T}_{\beta}=\sum_{\beta} j_{\beta} Q_{\beta}=\frac{j}{N} \boldsymbol{S}^{2}+\sum_{\beta}{ }^{\prime} j_{\beta} Q_{\beta} \\
& \geq \frac{j}{N} \boldsymbol{S}^{2}+j_{\min } \sum_{\beta}^{\prime} Q_{\beta} \\
& =\frac{j}{N} \boldsymbol{S}^{2}+j_{\min }\left(N-\frac{1}{N} \boldsymbol{S}^{2}\right)=\frac{j-j_{\min }}{N} \boldsymbol{S}^{2}+j_{\text {min }} N,
\end{aligned}
$$

using (48), the positivity of $Q_{\beta}$, and lemma 3 . The other inequality follows analogously.

We note that the proof does not depend on the dimension of spin space, but the optimal energy bounds may depend on this dimension. The regular pentagon is an example of a system where the above bounding parabolas are assumed by 3 -dimensional states, but not by 2-dimensional, i. e. , coplanar states, see section 6.3.1.

\section{Symmetric states}

In this section we will assume suitable symmetry properties of the spin system and correspondingly consider the notion of "symmetric states". We make use of some simple concepts and results of group theory, which may be found in many textbooks, e. g. [16].

Let $J_{\mu \nu}=0$ or $J$ and consider the graph $\Gamma=(\mathcal{V}, \mathcal{E})$ characterizing the spin system. Further let $\mathcal{G}$ denote the symmetry group of the graph $\Gamma$. Hence $\mathcal{G}$ consists of all permutations of $\mathcal{V}$ which map edges onto edges. $\mathcal{G}$ has a "natural" representation by real $N \times N$-matrices, by permuting the standard basis of $\mathbb{R}^{N}$ in the same way as the spin sites $\mu \in \mathcal{V}$. We will denote the $N \times N$-matrix representing any symmetry $g \in \mathcal{G}$ by $\mathbf{g}$ and by $\mathbb{G}$ the set of all $N \times N$-matrices obtained in this way. Obviously, 
Lemma 4 The adjacency matrix $\mathbb{J}$ commutes with all $\mathbf{g} \in \mathbb{G}$.

Hence

Lemma 5 The eigenspaces of $\mathbb{J}$ split into orthogonal direct sums of irreducible subrepresentations of the natural representation of $\mathcal{G}$.

The irreducible representations of the relevant groups are well known, see e. g. [16], and can easily be associated to the different (subspaces of) eigenspaces of $\mathbb{J}$.

For specific spin systems, e. g. magnetic molecules, the spin sites $\mu \in \mathcal{V}$ are embedded into the physical 3-space and the group $\mathcal{G}$ could also be identified with one of the point groups, i. e. finite subgroups of $\mathcal{O}(3, \mathbb{R})$. There are only a finite number of possibilities, see e. g. [16], 3.1.2. and 3.1.3. The most interesting cases are

- the dihedral group $\mathcal{D}_{n}$,

- the tetrahedral group $\mathcal{T}$,

- the octahedral group $\mathcal{O}$,

- the icosahedral group $\mathcal{Y}$,

as well as the improper point groups attached to them.

In what follows, we make the following general assumption:

Assumption 1 All exchange parameters $J_{\mu \nu}$ are 0 or $J$, and the group $\mathcal{G}$ operates transitively on $\mathcal{V}$.

The latter means that for each pair $\mu, \nu \in \mathcal{V}$ there is a permutation $g \in \mathcal{G}$ such that $g(\mu)=\nu$. The two groups $\mathcal{G}$ and $\mathcal{O}(3, \mathbb{R})$ operate independently on the set of states: If two matrices $\mathbf{g} \in \mathbb{G}$ and $R \in \mathcal{O}(3, \mathbb{R})$ are given, we define their action on a state $\boldsymbol{s}$ by matrix multiplication with $s$ which is again considered as an $N \times 3$-matrix:

Definition $2(R, \mathbf{g}) \bullet s \equiv R \mathbf{s g}^{-1}$.

The transformations $R \in \mathcal{O}(3, \mathbb{R})$ will also be referred to as "rotations/reflections in spin space".

We have the following obvious results:

Lemma 6 Let $\mathbf{g} \in \mathbb{G}$ and $R \in \mathcal{O}(3, \mathbb{R})$ and $\boldsymbol{s}$ be a state. Then

(i) $\boldsymbol{s}$ and $\mathrm{Rsg}^{-\mathbf{1}}$ have the same total spin length $S$,

(ii) If $\boldsymbol{s}$ is (weakly) stationary, then $\mathrm{Rs}^{-\mathbf{1}}$ is (weakly) stationary,

(iii) If $\boldsymbol{s}$ is weakly symmetric, then $\mathrm{Rs}^{-\mathbf{1}}$ is weakly symmetric,

(iv) if $\boldsymbol{s}$ is a (anti-) ground state, then $\mathrm{Rsg}^{-\mathbf{1}}$ is a (anti-) ground state.

Having defined the action of the product group $\mathcal{O}(3, \mathbb{R}) \times \mathbb{G}$ on states, it is natural to consider the corresponding subgroup leaving a given state invariant: 
Definition 3 For any state $\boldsymbol{s}$ let $\mathcal{G}_{\boldsymbol{s}} \equiv\left\{(R, \mathbf{g}) \in \mathcal{O}(3, \mathbb{R}) \times \mathbb{G} \mid R \boldsymbol{s} \mathbf{g}^{-1}=\boldsymbol{s}\right\} . \quad \mathcal{G}_{\boldsymbol{s}}$ is called the symmetry group of $\boldsymbol{s}$.

Definition $4 A$ state $s$ is called symmetric if the projection onto the second factor $\pi_{2}: \mathcal{G}_{\boldsymbol{s}} \longrightarrow \mathbb{G}$ is surjective, i. e. if for each $\mathbf{g} \in \mathbb{G}$ there exists a rotation/reflection $R \in \mathcal{O}(3, \mathbb{R})$ such that $R^{-1} \boldsymbol{s g}=\boldsymbol{s}$.

One may thus say that for symmetric states $s$ every permutation $\mathbf{g} \in \mathbb{G}$ of the vectors $\boldsymbol{s}_{\mu}$ can be compensated by a suitable rotation/reflection in spin space.

For example, if $(\mathcal{V}, \mathcal{E})$ is the graph of a regular $N$-polygon, the coplanar symmetric states are in 1 : 1-correspondence to the roots of unity, $z_{n}^{N}=1$, namely

$$
\boldsymbol{s}_{\mu}=z_{n}^{\mu}=\exp (i \mu n 2 \pi / N), \quad n, \mu=1, \ldots, N .
$$

Here $\mathcal{G}=\mathcal{D}_{N}$, the dihedral group of order $2 N$.

We have the following

Lemma 7 Let $\boldsymbol{s}_{(\mu)}^{(i)}$ be a non-vanishing $N \times 3$-matrix such that for all $\boldsymbol{g} \in \mathbb{G}$ there exists some $R \in \mathcal{O}(3, \mathbb{R})$ such that $\mathbf{s g}=R \boldsymbol{s}$. Then, for some suitable $\lambda \in \mathbb{R}$, $\lambda$ s will be a symmetric state.

Proof: It remains to show that $\lambda \boldsymbol{s}_{\mu}^{(i)}$ will be unit vectors for all $\mu=1, \ldots, N$. Fix some indices $\mu, \nu$. Using assumption 1 , we choose a $\boldsymbol{g} \in \mathbb{G}$ such that $(\boldsymbol{s g})_{\mu}=\boldsymbol{s}_{\nu}=R \boldsymbol{s}_{\mu}$. Since $R \in \mathcal{O}(3, \mathbb{R})$ the two vectors $\boldsymbol{s}_{\nu}$ and $\boldsymbol{s}_{\mu}$ have the same length. Because $\mu, \nu$ were arbitrarily chosen, all vectors $\boldsymbol{s}_{\mu}$ have the same length, say $\lambda^{-1}$, which completes the proof.

If a state $s$ is not collinear, then $R \boldsymbol{s}=\boldsymbol{s}$ implies $R=\mathbb{1}$. In this case for each $\mathbf{g} \in \pi_{2}\left(\mathcal{G}_{\boldsymbol{s}}\right)$ there exists a unique $R \in \mathcal{O}(3, \mathbb{R})$ such that $R^{-1} \boldsymbol{s g}=\boldsymbol{s}$. We will write $R=\rho_{\boldsymbol{s}}(\mathbf{g})$. It is easily shown that the map $\mathbf{g} \mapsto \rho_{\boldsymbol{s}}(\mathbf{g})$ is a linear representation of the subgroup $\pi_{2}\left(\mathcal{G}_{\boldsymbol{s}}\right) \subset \mathbb{G}$. If $\boldsymbol{s}$ is collinear we may uniquely fix $R$ by choosing $R \in\{\mathbb{1},-\mathbb{1}\}$ and thus obtain a representation $\rho_{\boldsymbol{s}}$ also in this case.

For the case of symmetric $\boldsymbol{s}$ we have $\pi_{2}\left(\mathcal{G}_{\boldsymbol{s}}\right)=\mathbb{G}$ and hence $\rho_{\boldsymbol{s}}$ will be an $n$ dimensional representation of $\mathbb{G}$ with $n \leq 3$.

In the case of a symmetric ground state it is thus necessary that the eigenspace of $\mathbb{J}$ corresponding to the smallest eigenvalue $j_{\text {min }}$ contains an irreducible representation of $\mathbb{G}$ of dimension $n \leq 3$. If it does not, we can exclude symmetric ground states.

For practical purposes of constructing (anti-)ground states it would be desirable to invert the process and to reconstruct $s$ from a given $n$-dimensional subrepresentation $\rho$ of the natural representation of $\mathcal{G}, n \leq 3$. We will now describe this procedure and consider, for sake of simplicity, only the case $n=3$. Let $\mathcal{S}$ be an 3-dimensional subspace 
of $\mathbb{R}^{N}$ left invariant by all $\boldsymbol{g} \in \mathbb{G}$ and $\boldsymbol{s}_{(\mu)}^{i}, i=1,2,3$ a basis of $\mathcal{S}$. It follows that the three vectors $\sum_{\mu} \boldsymbol{g}_{\mu(\nu)} \boldsymbol{s}_{\mu}^{i}, i=1,2,3$ are linear combinations of the basis vectors, hence

$$
\sum_{\mu} \boldsymbol{g}_{\mu \nu} \boldsymbol{s}_{\mu}^{i}=\sum_{j} \rho_{j}^{i}(\boldsymbol{g}) \boldsymbol{s}_{\nu}^{j}
$$

or, in matrix notation, $\rho(\boldsymbol{g}) \boldsymbol{s}=\boldsymbol{s} \boldsymbol{g}$. Being a real representation of a finite group, $\rho$ is equivalent to an orthogonal representation, i. e. $\widetilde{\rho}(\boldsymbol{g}) \equiv T \rho(\boldsymbol{g}) T^{-1}$ is orthogonal with some invertible $3 \times 3$-matrix $T$. It follows that

$$
\widetilde{\rho}(\boldsymbol{g})(T \boldsymbol{s})=(T \boldsymbol{s}) \boldsymbol{g}
$$

which means that $T \boldsymbol{s}$ is a symmetric state with $\rho_{T \boldsymbol{s}}=\rho$. Note that the orthogonality of the representation is crucial in order to obtain a row $(T s)_{\mu}^{(i)} \mu=1, \ldots, N$ of unit vectors, compare lemma 7 . Hence we have proven the following

Proposition 2 Let $\rho$ be a 3-dimensional subrepresentation of the natural representation of $\mathcal{G}$. Then there exists a 3-dimensional symmetrical state $\boldsymbol{s}$ such that $\rho_{\boldsymbol{s}}=\rho$.

Further there holds

Proposition 3 Each stationary symmetric state $\boldsymbol{s}$ is weakly symmetric.

Proof: We will use a matrix notation and write $\kappa \equiv \operatorname{diag}\left(\kappa_{1}, \ldots, \kappa_{N}\right)$. Then the condition (16) for stationarity may be written as $\boldsymbol{s} \mathbb{J}=\boldsymbol{s} \kappa$. For $\mathrm{g} \in \mathbb{G}$ we have $\widetilde{\kappa} \equiv$ $\mathbf{g}^{-1} \kappa \mathbf{g}=\operatorname{diag}\left(\kappa_{\mathbf{g}(1)}, \ldots, \kappa_{\mathbf{g}(N)}\right)$. We conclude $\boldsymbol{s} \kappa \mathbf{g}=\boldsymbol{s} \rrbracket \mathbf{g}=\boldsymbol{s} \mathbf{g} \mathbb{J}=\rho_{\boldsymbol{s}}(\mathbf{g}) \boldsymbol{s} \mathbb{J}=\rho_{\boldsymbol{s}}(\mathbf{g}) \boldsymbol{s} \kappa=$ $\boldsymbol{s} \mathbf{g}\left(\mathbf{g}^{-1} \kappa \mathbf{g}\right)=\rho_{\boldsymbol{s}}(\mathbf{g}) \boldsymbol{s} \widetilde{\kappa}$. Hence $\boldsymbol{s} \kappa=\boldsymbol{s} \widetilde{\kappa}$, which is in components $\kappa_{\mu} \boldsymbol{s}_{\mu}=\kappa_{\mathbf{g}(\mu)} \boldsymbol{s}_{\mu}$ whence $\kappa_{\mu}$ is independent of $\mu$ (using assumption 1 ).

The converse of proposition 3 is not true: There are ground states of the icosidodecahedron which are weakly symmetric, but not symmetric, see section 6.5. The condition of stationarity in proposition 3 can be replaced by another condition:

Definition 5 Let $\mathcal{G}_{\boldsymbol{s}}$ denote the subgroup of $\mathcal{G}$ leaving $\mu \in \mathcal{V}$ fixed and $\mathbb{G}(\mu)$ the matrix group generated by its natural representation. A symmetric state $\boldsymbol{s}$ will be called isotropic if $\rho_{\boldsymbol{s}}(\mathbb{G}(\mu))$ contains at least one rotation with an angle $\alpha \neq 0, \pi$.

Lemma 8 If $\boldsymbol{s}$ is isotropic and $\rho_{\boldsymbol{s}}=\rho_{\boldsymbol{s}^{\prime}}$ then $\boldsymbol{s}_{\mu}= \pm \boldsymbol{s}_{\mu}^{\prime}$.

Proof: $\boldsymbol{s}_{\mu}$ is invariant under all rotations/reflections $\rho_{\boldsymbol{s}}(\mathbf{g}), \mathbf{g} \in \mathbb{G}(\mu)$. Since some of these is a rotation with an angle $\alpha \neq 0, \pi$, the axis of rotation will be unique and $\rho_{\boldsymbol{s}}=\rho_{\boldsymbol{s}^{\prime}}$ implies $\boldsymbol{s}_{\mu}= \pm \boldsymbol{s}_{\mu}^{\prime}$.

Proposition 4 Each isotropic state is stationary, hence weakly symmetric.

Proof: Using the above matrix notation we obtain $\boldsymbol{s} \rrbracket \mathbf{g}=\boldsymbol{s} \mathbf{g} \mathbb{J}=\rho_{\boldsymbol{s}}(\mathbf{g}) \boldsymbol{s} \mathbb{J}$ hence $\boldsymbol{s} \mathbb{J}$ is a symmetric (not normalized) state with $\rho_{\boldsymbol{s} \rrbracket}=\rho_{\boldsymbol{s}}$. Using that $s$ is isotropic and lemma (8) we conclude $(\boldsymbol{s} \mathbb{J})_{\mu}= \pm \lambda \boldsymbol{s}_{\mu}$, hence $\boldsymbol{s}$ is stationary, and, by proposition (3), weakly symmetric. 


\section{Examples}

We will mainly consider $\mathrm{AF}$ systems and set $J>0$ throughout this section if not mentioned otherwise.

\subsection{The general spin triangle}

We consider spin systems with $N=3$ and general coupling coefficients $J_{1}, J_{2}$ and $J_{3}$. The special symmetric case $J_{1}=J_{2}=J_{3}$ is atypical and its properties are discussed later. Another special case is the 3 -chain with $J_{1}=0, J_{2}=J_{3}$, which is probably the simplest example of a non-parabolic system. Its investigation will be left for the reader.

Let $\mathbf{s}_{\nu}, \nu=1,2,3$, be any spin configuration and consider the $3 \times 3$-matrix $\mathcal{S}$ with coefficients

$$
\mathcal{S}_{\mu \nu}=\mathbf{s}_{\mu} \cdot \mathbf{s}_{\nu}
$$

It is positive and its diagonal consists of 1 's. Conversely, any $3 \times 3$-matrix with these properties is of the form (54). This follows from the spectral theorem. These matrices can be written in the form

$$
\mathcal{S}=\left(\begin{array}{ccc}
1 & w & v \\
w & 1 & u \\
v & u & 1
\end{array}\right) \equiv[u, v, w]
$$

where $u, v, w$ are real numbers subject to the constraint

$$
\operatorname{det} \mathcal{S}=1-\left(u^{2}+v^{2}+w^{2}\right)+2 u v w \geq 0, \text { and } u^{2}, v^{2}, w^{2} \leq 1 .
$$

Hence the set $\mathcal{P}$ of the matrices of the form (54) can be considered as a compact convex subset of $\mathbb{R}^{3}$. It has the form of an "inflated tetrahedron", see figure 1 . Since the map

$$
\begin{aligned}
\pi & : \mathcal{P} \longrightarrow \mathbb{R}^{2} \\
\pi[u, v, w] & \equiv\left(3+2(u+v+w), J_{1} u+J_{2} v+J_{3} w\right) \\
& =\left(S^{2}(\mathbf{s}), \frac{1}{2} H_{0}(\mathbf{s})\right)
\end{aligned}
$$

is affine, the set $\mathcal{P}_{2} \equiv \pi[\mathcal{P}]$ of all possible values of the energy vs. square of the total spin will be a compact convex set too. To simplify the geometry we will assume $J_{1}+J_{2}+J_{3}=0$. Then $\mathcal{P}_{2}$ can be considered as the orthogonal projection of $\mathcal{P}$ onto a suitable plane. If we introduce new coordinates in the $u, v, w$-space by

$$
\begin{aligned}
\sigma & =u+v+w \\
\epsilon & =J_{1} u+J_{2} v+J_{3} w \\
\tau & =\left(J_{2}-J_{3}\right) u+\left(J_{3}-J_{1}\right) v+\left(J_{1}-J_{2}\right) w
\end{aligned}
$$

the orthogonal projection $\pi$ is essentially the projection onto the $\sigma, \epsilon$-plane. Hence the boundary of $\mathcal{P}_{2}$ can be obtained by solving the two equations

$$
\operatorname{det} \mathcal{S}=0,
$$




$$
\nabla \operatorname{det} \mathcal{S} \cdot\left(\begin{array}{c}
J_{2}-J_{3} \\
J_{3}-J_{1} \\
J_{1}-J_{2}
\end{array}\right)=0 .
$$

The first equation (63) defines the boundary of $\mathcal{P}$, the second one (64) is satisfied by those points of the boundary of $\mathcal{P}$ which are orthogonally projected onto the boundary of $\mathcal{P}_{2}$. By using computer algebra software it is straightforward to express (63) and (64) as equations for $\sigma, \epsilon, \tau$ and to eliminate $\tau$ between (63) and (64). Yet the result for the general case is too complex to be reproduced here. We choose the special case

$$
J_{1}=0, J_{2}=1, J_{3}=-1
$$

and obtain the following equation for the boundary of $\mathcal{P}_{2}$

$$
\begin{aligned}
0= & S^{2}\left(9-10 S^{2}+S^{4}\right)^{2}+\left(-27+288 S^{2}+18 S^{4}-24 S^{6}+S^{8}\right) \epsilon^{2} \\
& -8\left(3+S^{2}\right)^{2} \epsilon^{4}+16 \epsilon^{6},
\end{aligned}
$$

where we have re-substituted $\frac{S^{2}-3}{2}$ for $\sigma$. The actual boundary of $\mathcal{P}_{2}$ is only a part of the family of curves defined by (66) and is shown in figure 2. Here we have an example where the curves $E_{\text {min }}(S)$ and $E_{\max }(S)$ can be explicitely calculated and are not parabolas, although they are close to their bounding parabolas which are represented as broken lines in figure 2. This is not in contradiction with theorem 11 since the ground state of this system is coplanar but has $S>0$.

We expect that similar figures also appear for the general system of $N$ spins. Parabolic systems are probably rare cases, some of which will be considered in the remaining subsections.

\section{2. $\mathcal{S}_{N}$-symmetric systems}

Recall that the $N$-pantahedron was defined as a system of $N$ spins where each pair $(\mu, \nu)$ interacts with equal strength $J>0$. These systems serve mainly as exactly solvable model examples for the sake of illustration, but the dimer, the triangle, and the tetrahedron are also of experimental relevance [17], [18], [19]. The symmetry group of the $N$-pantahedron is obviously the group $\mathcal{S}_{N}$ of all permutations of $N$ spin sites.

By completing squares the Hamiltonian can be written as

$$
H_{0}=J\left(\boldsymbol{S}^{2}-N\right) \text {. }
$$

Since the energy depends only on $S$, each state is a relative (anti-)ground state with

$$
E_{\text {min }}(S)=E_{\text {max }}(S)=J\left(S^{2}-N\right) .
$$

For $S=0$ there exist symmetrical ground states only if $N \leq 4$. These are the obvious dimer, triangle, and tetrahedron configurations in spin space. The dimension dim of the irreducible representations of $\mathcal{S}_{N}$ is given by the number of standard Young tableaus (see [16]) and hence $\operatorname{dim}=1$ or $\operatorname{dim} \geq N-1$. The one-dimensional representations are 
either the trivial one corresponding to the total anti-ground state or the one attaching to each permutation its sign, which is not generated by a symmetric state. Thus there are no symmetrical ground states for $N>4$. For example, a cyclic permutation of 3 spins leaving 2 other spins invariant cannot be compensated by a reflection/rotation in spin space.

The coplanar $N$-polygon configuration in spin space does however define a weakly symmetric ground state with $\kappa_{\mu}=-1$. Hence the construction of theorem 1 yields symmetrical relative ground states $\hat{\boldsymbol{s}}(S)$. We will check our equation (23) for this case: Let $\mathbb{E}$ denote the $N \times N$-matrix with $E_{\mu \nu}=1 / N$ for all $\mu, \nu$. It is the matrix of the projector onto the one-dimensional subspace spanned by the vector $\mathbf{1}=\frac{1}{\sqrt{N}}(1,1, \ldots, 1)$. The adjacency matrix for the $N$-pantahedron is $\mathbb{J}=J(N \mathbb{E}-\mathbb{1})$. Hence its eigenvalues are $j=J(N-1)$ with eigenvector $\mathbf{1}$ and $j_{\min }=-J$ with the $(N-1)$-dimensional eigenspace of vectors orthogonal to $\mathbf{1}$. Thus $j_{\min }=j_{\operatorname{maxi}}$ and the two functions $E_{\min }(S)$ and $E_{\text {max }}(S)$ coincide in accordance with what has been said above.

\section{3. $\mathcal{D}_{N}$-symmetric systems}

6.3.1. The $N$-polygon The $N$-polygon has the dihedral group $\mathcal{D}_{N}$ as symmetry group. We have already mentioned the set of $S=0$, stationary, coplanar, symmetric states $s$ given by

$$
\boldsymbol{s}_{\mu}=z_{n}^{\mu}, \quad \mu=0, \ldots, N-1,
$$

where $z_{n}=\exp (i n 2 \pi / N), n=1, \ldots, N$, is a root of unity, $z_{n}^{N}=1$. Indeed, these states are the complex eigenvectors of the corresponding adjacency matrix

$$
\mathbb{J}=J\left(\begin{array}{ccccccc}
0 & 1 & 0 & \ldots & \ldots & 0 & 1 \\
1 & 0 & 1 & 0 & \ldots & \ldots & 0 \\
0 & 1 & 0 & 1 & 0 & \ldots & 0 \\
\vdots & & & & & & \\
0 & \ldots & \ldots & 0 & 1 & 0 & 1 \\
1 & 0 & \ldots & \ldots & 0 & 1 & 0
\end{array}\right)
$$

The eigenvalues are obtained by

$$
(\mathbb{J} s)_{\mu}=J\left(z_{n}^{\mu+1}+z_{n}^{\mu-1}\right)=2 J \cos (2 \pi n / N) \boldsymbol{s}_{\mu}
$$

and the corresponding energies are

$$
E_{n}=2 J N \cos (2 \pi n / N), \quad n=0, \ldots, N-1 .
$$

It follows that

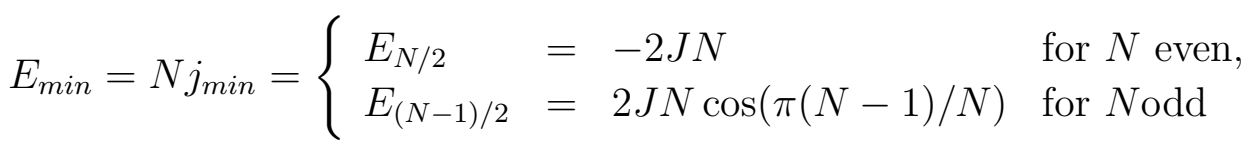

and

$$
E_{\text {max }}=E_{0}=N j=2 N J
$$


This follows since the bounds of lemma 1 are attained by these states. For $N=5$ the ground state of the pentagon may be vizualized as a pentagram in spin space and analogously for other odd $N$.

Moreover,

$$
E_{\text {max }}(0)=E_{1}=N j_{\max i}=2 N J \cos (2 \pi / N) .
$$

The relative ground states of theorem 1 have energies

$$
E_{\text {min }}(S)= \begin{cases}2 J\left(2 \frac{S^{2}}{N}-N\right) & \text { for } N \text { even } \\ 2 J\left(\left(1-\cos \left(\pi \frac{N-1}{N}\right)\right) \frac{S^{2}}{N}+N \cos \left(\pi \frac{N-1}{N}\right)\right) & \text { for } N \text { odd }\end{cases}
$$

and

$$
E_{\max }(S)=2 J\left(\left(1-\cos (2 \pi / N) \frac{S^{2}}{N}+N \cos (2 \pi / N)\right)\right.
$$

Both parabolas meet in the anti-ground state with $E_{\max }=2 J N$ for $S=N$.

6.3.2. Coplanar states of the pentagon The regular pentagon as a special case dealt with in the preceding subsection assumes its bounding parabolas. However, the extremal energies $E_{\min }(S)$ and $E_{\max }(S)$ can only be realized by non-coplanar states, except for $S=0$ and $S=5$. This has not been rigorously proven but shown by numerical evidence, see figure 3. The spectrum $E$ versus $S^{2}$ realized by coplanar states is a subset of the full spectrum with concave boundaries $E_{\min }^{\text {coplanar }}(S)$ and $E_{\text {max }}^{\text {coplanar }}(S)$.

The permutation of spin sites $(0 \leftrightarrow 0,1 \rightarrow 2 \rightarrow 4 \rightarrow 3 \rightarrow 1)$ leaves the Hamiltonian invariant and maps relative ground states onto anti-ground states, hence $E_{\text {min }}^{\text {coplanar }}(S)=S^{2}-5-E_{\max }^{\text {coplanar }}(S)$. The (anti-) ground state with $S=1$ and $E=-7$ (resp. $E=3$ ) is especially remarkable: Its state vectors $\boldsymbol{s}_{\mu}$ occupy the vertices of a regular hexagon leaving one vertex free. The anti-ground state $\left(E_{\max }^{\text {coplanar }}(1)=3\right)$ satisfies equation (16) with $\chi=0$, hence it is a stationary state, not only weakly stationary as other relative anti-ground states. Actually, $E_{\max }^{\text {coplanar }}$ has a local minimum at $S=1$, see figure 3. For axisymmetric weakly stationary states in the neighborhood of the hexagonal state, equation (16) can be solved analytically by using computer algebra software and hence $E$ and $S^{2}$ can be expressed as functions of a common parameter, although of forbidding complexity. The resulting curve turns out to be a part of the upper boundary of the coplanar spectrum and is displayed in figure 3, as well as the corresponding lower boundary part.

6.3.3. The pentagonal star We obtain the pentagonal star $(N=6)$ by joining the 5 vertices of the pentagon with its mid-point, see figure 4 . This system has $\mathcal{D}_{5}$ symmetry, but it is not $\mathcal{D}_{5}$-symmetric in our sense, since $\mathcal{D}_{5}$ does not operate transitively on the 6 spin sites. Nevertheless, we will discuss this example since it has the interesting property that its ground states are not coplanar and $E_{\min }(S), E_{\max }(S)$ are only piecewise parabolic. 
If we add to the 10 edges of the pentagonal star the 5 edges of the corresponding pentagram we obtain the 15 edges of the 6-pantahedron. Hence its Hamiltonian reads

$$
H=S^{2}-6-H_{5},
$$

where $H_{5}$ is the Hamiltonian of the pentagram, which is the same as that for the pentagon, up to a suitable permutation of the spin sites. This shows that the configurations minimizing $H$ for given $S$ are exactly those which maximize $H_{5}$, analogously for $E_{\max }(S)$. The maximal values for $H_{5}$ are given by the parabola, compare (77),

$$
E_{\text {max }}^{(5)}\left(S_{5}\right)=\frac{2(1-\cos (2 \pi / 5))}{5} S_{5}^{2}+10 \cos (2 \pi / 5),
$$

where $S_{5}$ is the length of the total spin of the 5 vertices of the pentagon. Here $S_{5}$ has to be chosen maximal for given $S$, that means that $S_{5}=S+1$ for $0 \leq S \leq 4$ and $S_{5}=5$ for $4 \leq S \leq 6$. This yields

$$
E_{\text {min }}(S)=\left\{\begin{array}{lll}
\frac{5+\sqrt{5}}{10}(S-4)(S+1+\sqrt{5}) & \text { if } & 0 \leq S \leq 4 \\
S^{2}-16 & \text { if } \quad 4 \leq S \leq 6
\end{array}\right.
$$

Hence the total ground state is attained not for $S=0$, but for $S=\frac{3-\sqrt{5}}{2}=0.381966 \ldots$ with $E_{\min }=-5-2 \sqrt{5}=-9.47214 \ldots$. The corresponding spin configuration is shown in figure 5 .

It remains to show that no other, coplanar ground state exists. Employing the numerical result of the preceding subsection for the pentagon, $E_{\max }^{\text {coplanar }}(S)<E_{\max }(S)$ for $0<S<5$, it is easily shown that all coplanar states with $S=\frac{3-\sqrt{5}}{2}$ have an energy larger than $E_{\text {min }}$. The minimal energy for all coplanar states appears to be $E_{\text {min }}^{\text {coplanar }}=-9$, realized by a hexagonal ground state with $S=0$, but we do not yet have a rigorous proof for this claim.

Analogously one can show that

$$
E_{\text {max }}(S)= \begin{cases}\frac{5-\sqrt{5}}{10}(S+4)(S-1+\sqrt{5}) & \text { if } 1 \leq S \leq 6 \\ S^{2}-16+\frac{5(1+\sqrt{5})}{2} & \text { if } \quad 0 \leq S \leq 1\end{cases}
$$

\subsection{O-symmetric systems}

6.4.1. The cube As already mentioned the cube allows a bi-partition and hence possesses a ground state of the form

$$
\boldsymbol{s}_{\mu}=(-1)^{\mu} \boldsymbol{e}, \quad \mu=1, \ldots, 8,
$$

if the vertices are suitably labelled. From this we obtain relative ground states with

$$
E_{\text {min }}(S)=J\left(\frac{3}{4} S^{2}-24\right)
$$

since $j=3 J, j_{\text {min }}=-3 J$. Apart from $j$ and $j_{\text {min }}$ the other eigenvalues of $\mathbb{J}$ are $\pm J$ with three-fold degeneracy, respectively. The corresponding eigenspaces carry two inequivalent irreducible representations of $\mathcal{O}$, called $F_{1}, F_{2}$, see [16]. According to the 
considerations in section 5 we may conjecture that these two representations are generated by stationary, symmetric, non-coplanar states.

Indeed, this can be directly verified for the following states: Let $\boldsymbol{r}_{\mu}, \mu=1, \ldots, 8$ denote the unit vectors pointing to the vertices of the cube. The the states

$$
\boldsymbol{s}_{\mu}^{(1)} \equiv \boldsymbol{r}_{\mu}, \quad \mu=1, \ldots, 8,
$$

and

$$
\boldsymbol{s}_{\mu}^{(-1)} \equiv(-1)^{\mu} \boldsymbol{r}_{\mu}, \quad \mu=1, \ldots, 8,
$$

have the desired properties. They are easily vizualized: $\boldsymbol{s}^{(1)}$ is just the "cube in spin space" and $\boldsymbol{s}^{(-1)}$ the tetrahedron where each of the four distinct spin vectors is attached to pairs of vertices of the cube connected by space diagonals. $\boldsymbol{s}^{(1)}$ is the anti-ground state for $S=0$ with energy $E_{\max }(0)=8 \mathrm{~J}$. Since it is not coplanar, theorem 1 is not directly applicable. However, it is possible to project the cube $\boldsymbol{s}^{(1)}$ onto a square and, in a second step, to enlarge the square to a square of unit vectors. Since these are linear transformations, the resulting co-planar state $\boldsymbol{s}^{(1) \prime}$ is weakly symmetric and has the same energy as before, namely $8 J=E_{\max }(0)$. Now theorem 1 yields $E_{\max }(S)=J\left(\frac{1}{4} S^{2}+8\right)$.

6.4.2. The octahedron As noted in [9], the octahedron can be decomposed into four triangles with disjoint edges and it is 3-colorable. Hence it has coplanar ground states with $S=0$ which are obtained by extensions of the local ground states of the triangles. Since $j=4 J$ and $j_{\min }=-2 J$ we again obtain the RBS-parabola

$$
E_{\text {min }}(S)=J\left(S^{2}-12\right)
$$

The eigenspace of $\mathbb{J}$ corresponding to $j_{\min }$ is two-dimensional and carries a real, irreducible representation of $\mathcal{O}$. This corresponds to the one-dimensional complex eigenspace of $\mathbb{I}$ spanned by the vector

$$
\boldsymbol{s}_{\mu}=z_{n(\mu)}, \quad \mu=1, \ldots, 6,
$$

where the

$$
z_{n} \equiv \exp (i n 2 \pi / 3), \quad n=0,1,2,
$$

are the 3rd roots of unity and $\mu \mapsto n(\mu)$ denotes any 3-coloring of the octahedron. It follows that the state $s$ is symmetrical.

The remaining 3 -dimensional eigenspace of $\mathbb{J}$ with eigenvalue $j_{\text {maxi }}=0$ carries the 3-dimensional self-representation of $\mathcal{O}$ and hence corresponds to the symmetric antiground state with $S=0$ which can be vizualized as a octahedron in spin space. Its energy is $E_{\max }=0$. Again, as in the case of the cube, there is a weakly symmetric co-planar state with the same energy and theorem 1 yields $E_{\max }(S)=\frac{2 J}{3} S^{2}$. This state has the form of a (not necessarily regular) hexagon such that opposing vertices of the octahedron in real space are mapped onto opposing vertices of the hexagon in spin space. 
Table 1. Eigenvalues $j_{n}$ of the adjacency matrix $\mathbb{J}$ of the cuboctahedron (1st column) together with their degeneracy (2nd column). In the 3rd column the irreducible representations of $\mathcal{O}$ are indicated which occur in the corresponding eigenspaces. The nomenclature $A_{1}, A_{2}, E, F_{1}, F_{2}$ follows [16].

\begin{tabular}{rll}
\hline$j_{n} / J$ & degeneracy & irreducible representations of $\mathcal{O}$ \\
\hline-2 & 5 & $E \oplus F_{2}$ \\
0 & 3 & $F_{2}$ \\
2 & 3 & $F_{1}$ \\
4 & 1 & $A_{1}$ \\
\hline
\end{tabular}

The above RBS energy bounds can also be obtained more simply: Since $H_{0}=$ $\boldsymbol{S}^{2}-\left(\boldsymbol{S}_{16}^{2}+\boldsymbol{S}_{25}^{2}+\boldsymbol{S}_{34}^{2}\right)$, where $\boldsymbol{S}_{i j} \equiv \boldsymbol{S}_{i}+\boldsymbol{S}_{j}$ and $J=1$, the minimal energy $E_{\text {min }}(S)$ is obtained for $S_{i j}=2$ as $E_{\min }(S)=S^{2}-12$. Similarly the energy is maximal for $S_{i j}=\frac{1}{3} S$ which yields $E_{\max }(S)=S^{2}\left(1-\frac{3}{9}\right)=\frac{2}{3} S^{2}$.

6.4.3. The cuboctahedron The cuboctahedron is the quasi-regular polyhedron obtained by joining the mid-points of the cube's edges with their nearest neighbors, see [7], [8], and figure 6 .

It can be decomposed into 8 triangles with disjoint edges and it is 3-colorable, see figure 2. Hence there is a coplanar ground state with $S=0$ giving rise to an RBSparabola

$$
E_{\text {min }}(S)=J\left(\frac{1}{2} S^{2}-24\right),
$$

since $j=4$ and $j_{\min }=-2$. The eigenvalues of $\mathbb{J}$ together with their degeneracy and irreducible representations of $\mathcal{O}$ are summarized in table 1.

This has been calculated by using the formula connecting the characters with the multiplicity of irreducible representations, c. f. [16], 4.2.31b. From this table it is obvious that the trivial irreducible representation $A_{1}$ corresponds to the total anti-ground state, $E$ is spanned by the complex eigenvector of the coplanar ground state, and the self-representation $F_{1}$ corresponds to the cuboctahedron in spin space with the energy $E_{\max }(0)=12 j_{\operatorname{maxi}}=24 \mathrm{~J}$. It remains to identify the symmetrical states which generate the two 3 -dimensional irreducible representations $F_{2}$ corresponding to the eigenvalues 0 and -2 of $\mathbb{J}$.

The vertex vectors $\boldsymbol{r}_{\mu}, \mu=1, \ldots, 12$ of the cuboctahedron may be represented, up to normalization, by integer 3 -vectors with components $-1,0,1$ and exactly one 0 component. Then a state vector $\boldsymbol{s}_{\mu}$ may be defined by the following rule: Invert the first component of $\boldsymbol{r}_{\mu}$ after 0 and set the other two components to 0. Here "after" is understood cyclically, e. g. $(1,1,0) \mapsto(-1,0,0)$. Thus we obtain a state, see figure 7 where the spins $\boldsymbol{s}_{\mu}$ of adjacent vertices are orthogonal. Indeed, this state corresponds to the 3 -dimensional eigenstate of $\mathbb{J}$ with eigenvalue 0 which is transformed under $\mathcal{O}$ 
according to $F_{2}$.

A second state $\boldsymbol{s}^{\prime}$ is obtained by a permutation of the $\boldsymbol{r}_{\mu}$. Each $\boldsymbol{r}_{\mu}$ is mapped onto that vertex where the above-defined state vector $\boldsymbol{s}_{\mu}$ points to:

$$
\boldsymbol{s}^{\prime}{ }_{\mu} \equiv 2 \boldsymbol{s}_{\mu}-\boldsymbol{r}_{\mu}, \quad \text { (not normalized) }
$$

Alternatively, $\boldsymbol{s}^{\prime}{ }_{\mu}$ is obtained from $\boldsymbol{r}_{\mu}$ by the following rule: Invert the first component of $\boldsymbol{r}_{\mu}$ after 0 and leave the other unchanged. For example, $(1,1,0) \mapsto(-1,1,0)$. Also this state generates a 3 -dimensional subspace of the eigenspace of $\mathbb{J}$ with $j_{\text {min }}=-2 J$ which transforms under $\mathcal{O}$ according to $F_{2}$.

Geometrically, $\boldsymbol{s}^{\prime}$ is a figure in spin space formed by four Stars of David, see figure 8. Thus the cuboctahedron has a coplanar ground state with $S=0$ as well as a noncoplanar one with the same energy.

\section{5. $\mathcal{Y}$-symmetrical systems}

We will consider the two remaining Platonic solids as well as the quasi-regular icosidodecahedron and again calculate the decomposition of the eigenspaces of the adjacency matrix $\mathbb{J}$ into $\mathcal{Y}$-irreducible subspaces for these three cases. As in the case of $\mathcal{O}$-symmetric systems, the eigenvalue $j_{\text {maxi }}$ always corresponds to the 3 -dimensional self-representation of $\mathcal{Y}$ called $F_{1}$. However, we have not found coplanar states realizing $j_{\operatorname{maxi}}$ and hence the question whether $E_{\max }(S)$ will be a parabola remains open.

The situation for ground states is different from the previous examples: For the icosahedron and the dodecahedron we find symmetric ground states with $S=0$ corresponding to the 3 -dimensional irreducible representation $F_{2}$ of $\mathcal{Y}$, but no coplanar ones. Moreover, numerical calculations yield the results

$$
\begin{aligned}
& E_{\text {min }}^{\text {coplanar }} \approx-43.0614 \ldots J>E_{\text {min }}=-20 \sqrt{5} J \text { for the dodecahedron, } \\
& E_{\text {min }}^{\text {coplanar }} \approx-24 J \quad>E_{\text {min }}=-12 \sqrt{5} J \text { for the icosahedron. }
\end{aligned}
$$

Hence we conjecture that there are no coplanar ground states and hence, according to theorem $2, E_{\min }(S)$ will not be an exact parabola. Of course, even overwhelming numerical evidence cannot be considered as a rigorous proof. We can only strictly exclude symmetric coplanar ground states, since there are no two-dimensional irreducible repre-

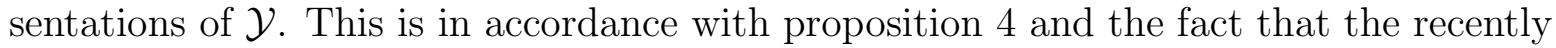
discovered $S=0$ ground states of the icosidodecahedron 9 are coplanar and weakly symmetric, but not symmetric.

For the two $\mathcal{Y}$-symmetrical Platonic solids we will investigate more closely the 3-dimensional geometry of the ground states. By numerical simulation of a heat bath at zero temperature, Ch. Schröder [20] has found the angles between adjacent spins to be $\alpha_{I} \approx 116.6^{\circ}$ for the icosahedron and $\alpha_{D} \approx 138.2^{\circ}$ for the dodecahedron. 
Table 2. Eigenvalues $j_{n}$ of the adjacency matrix $\mathbb{J}$ of the icosahedron (1st column) together with their degeneracy (2nd column). In the 3rd column the irreducible representations of $\mathcal{Y}$ are indicated which occur in the corresponding eigenspaces. The nomenclature $A, F_{1}, F_{2}, G, H$ follows 16 .

\begin{tabular}{rll}
\hline$j_{n} / J$ & degeneracy & irreducible representations of $\mathcal{Y}$ \\
\hline$-\sqrt{5}$ & 3 & $F_{2}$ \\
-1 & 5 & $H$ \\
$\sqrt{5}$ & 3 & $F_{1}$ \\
5 & 1 & $A$ \\
\hline
\end{tabular}

Table 3. Eigenvalues $j_{n}$ of the adjacency matrix $\mathbb{J}$ of the dodecahedron (1st column) together with their degeneracy (2nd column). In the 3rd column the irreducible representations of $\mathcal{Y}$ are indicated which occur in the corresponding eigenspaces. The nomenclature $A, F_{1}, F_{2}, G, H$ follows 16 .

\begin{tabular}{rll}
\hline$j_{n} / J$ & degeneracy & irreducible representations of $\mathcal{Y}$ \\
\hline$-\sqrt{5}$ & 3 & $F_{2}$ \\
-2 & 4 & $G$ \\
0 & 4 & $G$ \\
1 & 5 & $H$ \\
$\sqrt{5}$ & 3 & $F_{1}$ \\
3 & 1 & $A$ \\
\hline
\end{tabular}

From the knowledge of the corresponding irreducible subrepresentation of the natural representation of $\mathcal{Y}$ in $\mathbb{R}^{N}$ we can now exactly determine the symmetric ground state, see proposition 2. It turns out that these states are well-known stellated geometrical structures, called "Great Icosahedron" for the ground state of the icosahedron and "Great Stellated Dodecahedron" for the ground state of the dodecahedron, see [7] and [8]. Thus the angles between adjacent spins are just the angles between neighboring vertices of these stellated structures and hence have the exact values

$$
\alpha_{I}=\arccos \left(-\frac{\sqrt{5}}{5}\right), \alpha_{D}=\arccos \left(-\frac{\sqrt{5}}{3}\right),
$$

in agreement with the numerical findings of [20].

\section{Summary}

We have tried to give a systematic survey of the properties of (relative) ground states of classical Heisenberg spin systems with particular emphasis on symmetric systems. Further we have devised various methods of ground state construction, e. g. extension of local ground states, construction of symmetric ground states from irreducible representations of the system's symmetry group, and the construction of relative (anti-) ground states from coplanar ground states with $S=0$. The latter procedure yields upper and lower parabolas as the boundaries of the $S$-resolved energy spectrum. Thus we have a 
Table 4. Eigenvalues $j_{n}$ of the adjacency matrix $\mathbb{J}$ of the icosidodecahedron (1st column) together with their degeneracy (2nd column). In the 3 rd column the irreducible representations of $\mathcal{Y}$ are indicated which occur in the corresponding eigenspaces. The nomenclature $A, F_{1}, F_{2}, G, H$ follows 16.

\begin{tabular}{rll}
\hline$j_{n} / J$ & degeneracy & irreducible representations of $\mathcal{Y}$ \\
\hline-2 & 10 & $H \oplus H$ \\
$1-\sqrt{5}$ & 3 & $F_{2}$ \\
-1 & 4 & $G$ \\
1 & 4 & $G$ \\
2 & 5 & $H$ \\
$1+\sqrt{5}$ & 3 & $F_{1}$ \\
4 & 1 & $A$ \\
\hline
\end{tabular}

sufficient condition for a system to be parabolic. Moreover, under certain assumptions, this condition can also be proved to be necessary.

The above issues are also relevant for the quantum theory of Heisenberg spin systems. It has been shown for various cases [10, 21] that the shape of the $S$-resolved energy spectrum of the quantum system is very well approximated by the curves $E_{\min }(S)$ and $E_{\text {max }}(S)$ of the corresponding classical system, if only the individual spin quantum number $s$ exceeds some moderate value, say $s=2$. Hence one can predict semi-quantitative features of the quantum spectrum if one knows the classical spectrum and $s \gtrsim 2$. For some quantum systems like the icosidodecahedron with $s=5 / 2$, the diagonalization of the Heisenberg Hamiltonian is totally impractical, whereas the boundary of the spectrum of the corresponding (parabolic) classical system is well-known. For other classical systems with the same symmetry group $\mathcal{Y}$, viz. the dodecahedron and the icosahedron we expect non-parabolicity, although the corresponding quantum systems may exhibit approximate rotational bands.

In this sense our results and case studies are to a great extent relevant also for real, quantum spin systems.

\section{Acknowledgements}

H.-J. S. would like to thank members of the Condensed Matter Physics Group of the Ames Laboratory for their warm hospitality during a visit when a part of this work was performed. Likewise, M. L. thanks members of Fachbereich Physik, Universität Osnabrück for their warm hospitality when the final version of the manuscript was completed. We also acknowledge the financial support of a travel grant awarded jointly by NSF-DAAD which facilitated these visits. Finally, it is a pleasure to thank M. Axenovich, K. Bärwinkel, J. Schnack, and Ch. Schröder for stimulating and helpful discussions. Ames Laboratory is operated for the United States Department of Energy 
by Iowa State University under Contract No. W-7405-Eng-82.

\section{References}

[1] D. Gatteschi, Adv. Mater. 6, p. 635 (1994)

[2] K. L. Taft, C. D. Delfts, G. C. Papaefthymiou, S. Foner, D. Gatteschi, S. J. Lippard, J. Am. Chem. Soc. 116, p. 823 (1994)

[3] A. Müller, F. Peters, M. T. Pope, and D. Gatteschi, Chem. Rev. 98, p. 239 (1998)

[4] A. Müller, S. Sarkar, S. Q. N. Shah, H. Bögge, M. Schmidtmann, Sh. Sarkar, P. Kögerler, B. Hauptfleisch, A. X. Trautwein, V. Schünemann, Angew. Chem. Int. Ed. 38, p. 3238 (1999)

[5] E. F. Bertaut, Spin Configurations of Ionic Structures: Theory and Practice, in: G. T. Rado, H. Suhl (eds.) Magnetism, Vol. III, pp. 150-209, Academic Press, New York (1963)

[6] D. H. Lyons, T. A. Kaplan, Phys. Rev. 120, p. 1580, (1960)

[7] H. S. M. Coxeter, Regular polytopes, Dover, New York (1973)

[8] http://mathworld.wolfram.com/topics/Polyhedra.htm

[9] M. Axenovich, M. Luban, Phys. Rev. B63, 100407 (2001)

[10] J. Schnack, M. Luban, Phys. Rev. B63, 014418 (2001)

[11] R. Diestel, Graph Theory, Springer, New York (1997)

[12] D. Cvetković, M. Doob, I. Gutman, A. Torĝasev, Recent results in the Theory of graph spectra, North-Holland, Amsterdam (1988)

[13] R. Abraham, J. E. Marsden, T. Ratiu, Manifolds, Tensor Analysis, and Applications, AddisonWesley, London (1983)

[14] K. Bärwinkel, H.-J. Schmidt, J. Schnack, Energy bounds for $n$-partite spin systems, in preparation

[15] H.-J. Schmidt, J. Schnack, M. Luban, Europhys. Lett. 55 (1), p. 105 (2001)

[16] W. Ludwig, C. Falter, Symmetries in physics, Springer, Berlin (1988)

[17] P. C. Junk, B. J. McCool, B. Moubaraki, K. S. Murray, and L. Spiccia, Angew. Chem. 111, Nr. 15, p. $2363(1999)$

[18] A. Müller, J. Meyer, A. Stammler, and A. Botar, Chem. Eur. J. 4, p. 1388 (1998)

[19] Y. Furukawa, M. Luban, F. Borsa, D. C. Johnston, A. H. Mahajan, L. L. Miller, A. Bino, D. Mentrup, and J. Schnack, Phys. Rev. B61, p. 8635 (2000)

[20] C. Schröder, private communication

[21] J. Schnack, private communication 


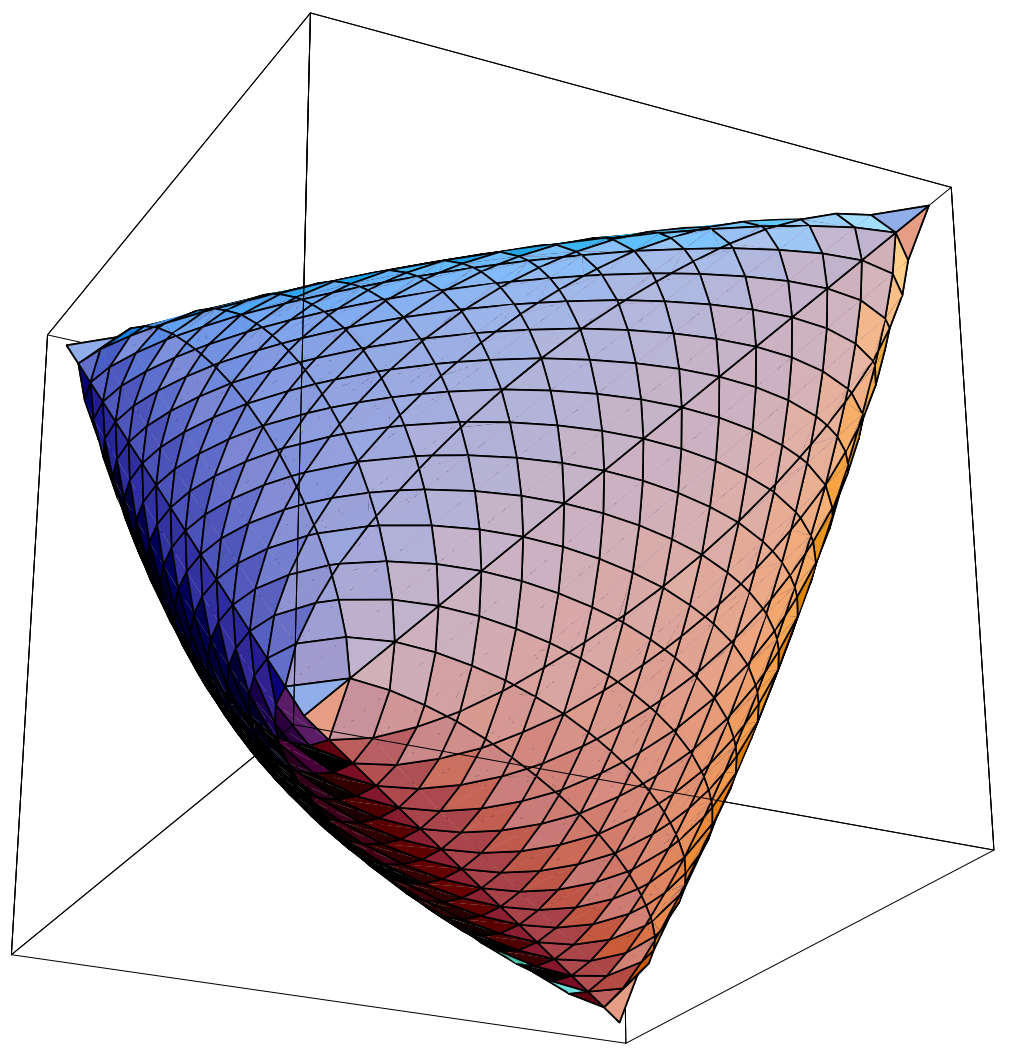

Figure 1. The 3-dimensional convex set $\mathcal{P}$ defined in (55) as seen from the view point $(4,-2,-2)$. Its shape is essentially identical with that shown in figure 2 .

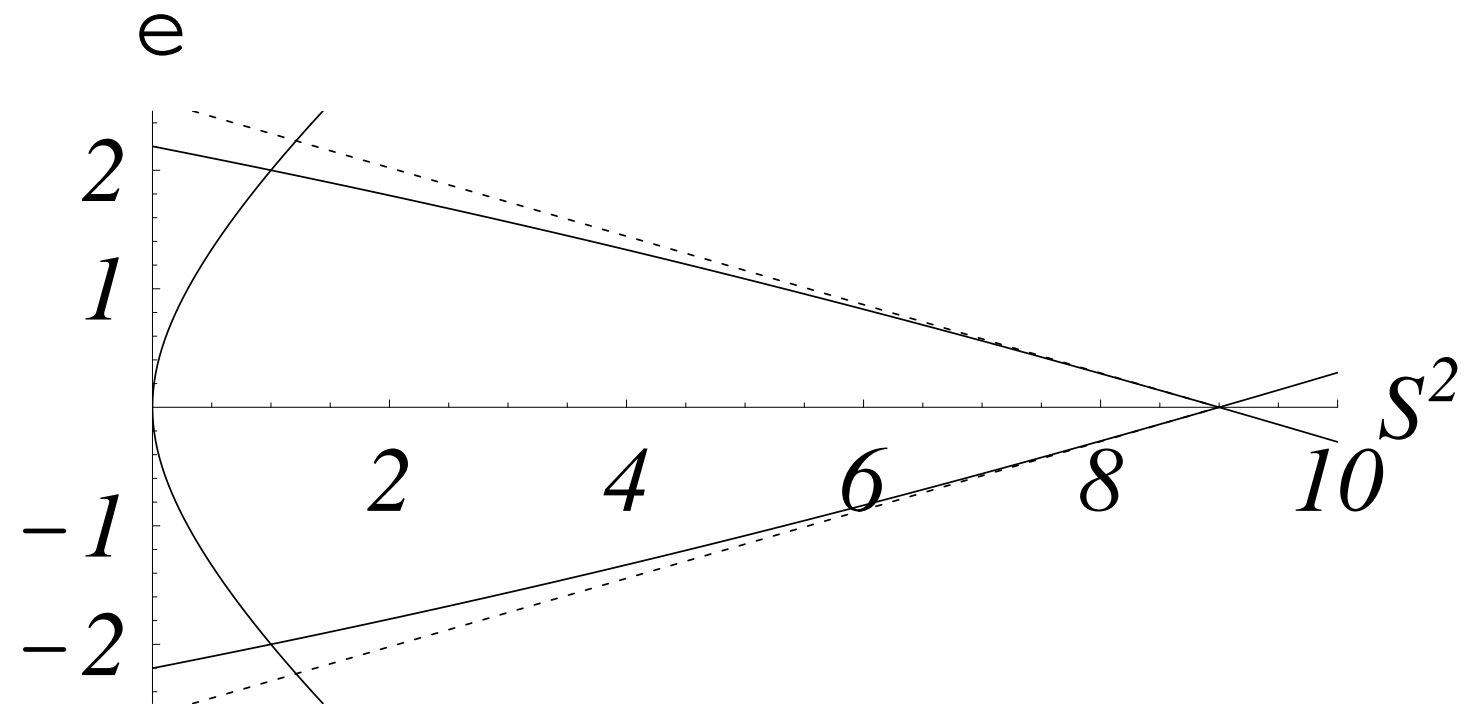

Figure 2. Energy $\epsilon$ versus square of total spin, $S^{2}$, for the triangle with $J_{1}=0, J_{2}=$ $1, J_{3}=-1$ according to (66). The broken lines represent the bounding parabolas of theorem 3. 


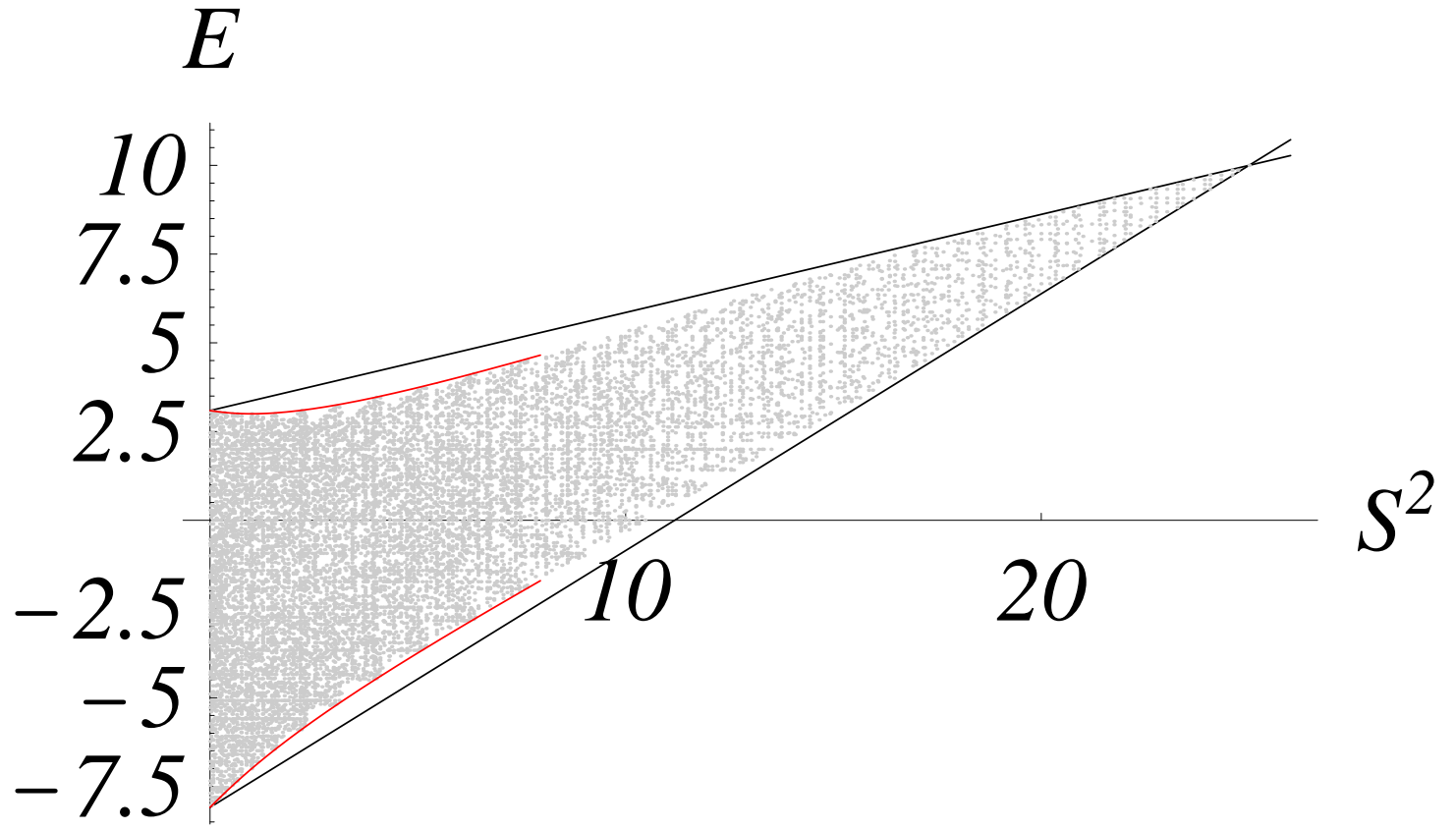

Figure 3. The shaded region represents the spectrum $E$ versus $S^{2}$ of coplanar states of the pentagon as determined by numerical methods. The straight lines are the boundaries of the full spectrum. Also a part of the boundary of the coplanar spectrum which can be analytically calculated is displayed.

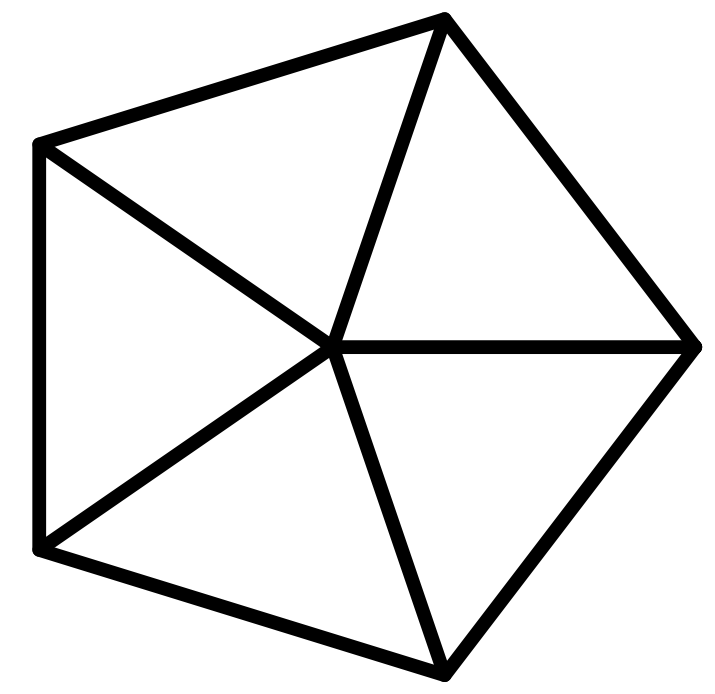

Figure 4. Pentagonal star as an example of a spin system without coplanar ground states. 


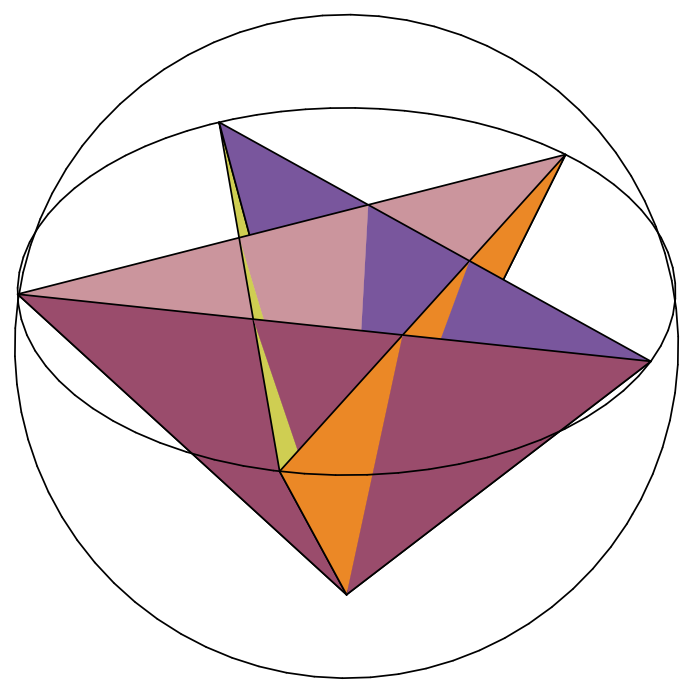

Figure 5. 3-dimensional ground state of the pentagonal star: The vertices correspond to 6 unit vectors in spin space and the edges correspond to the 10 bonds of the pentagonal star of figure 4 , see the remarks after (10). 


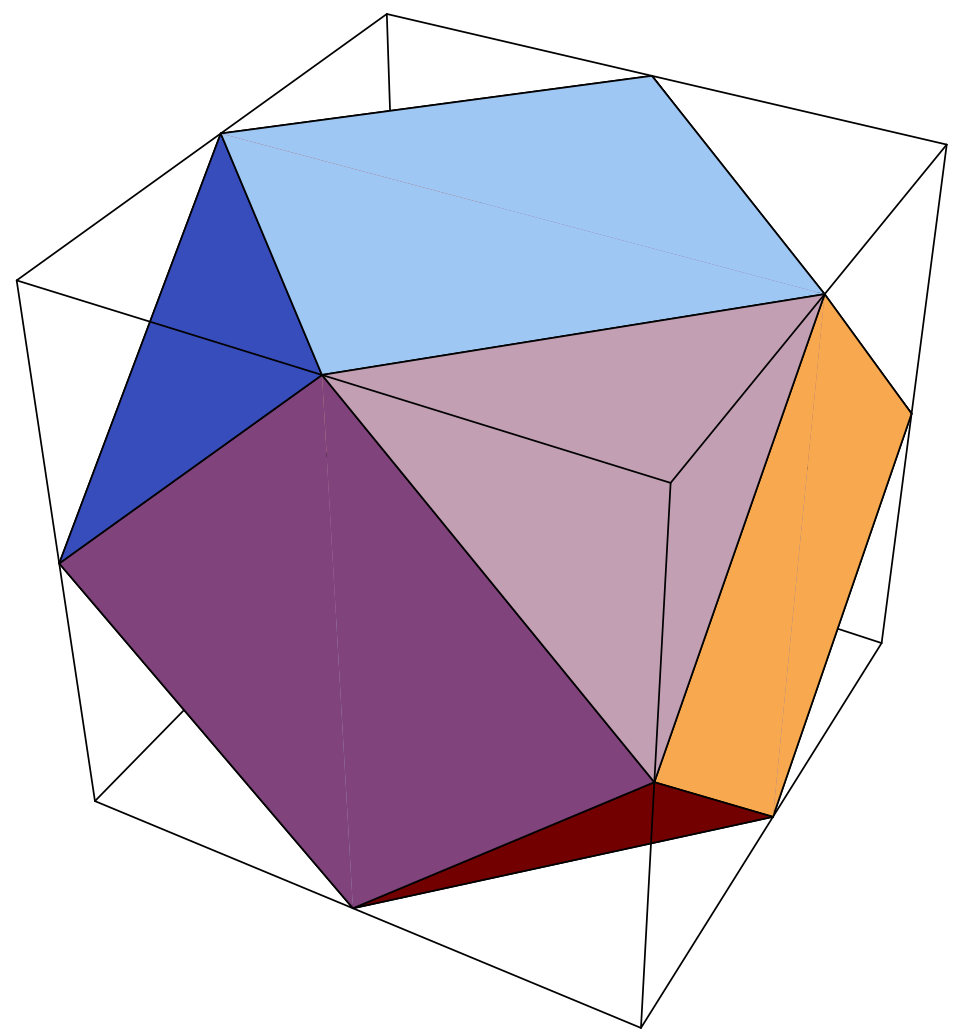

Figure 6. The cuboctahedron (shaded figure) is obtained by joining the mid-points of the cube's edges with their nearest neighbors. 


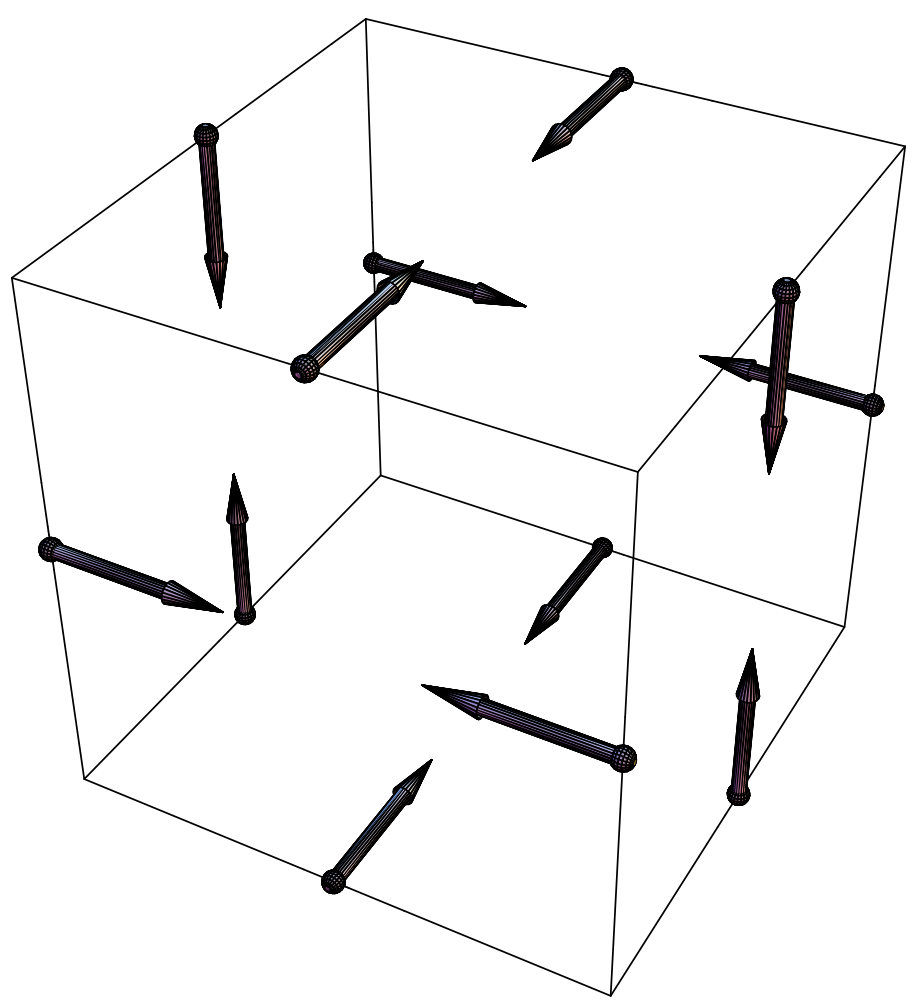

Figure 7. A stationary state of the cuboctahedron with energy $E=0$. The spin vectors $s_{\mu}$ are shown as small arrows attached to vertices of the cuboctahedron. 


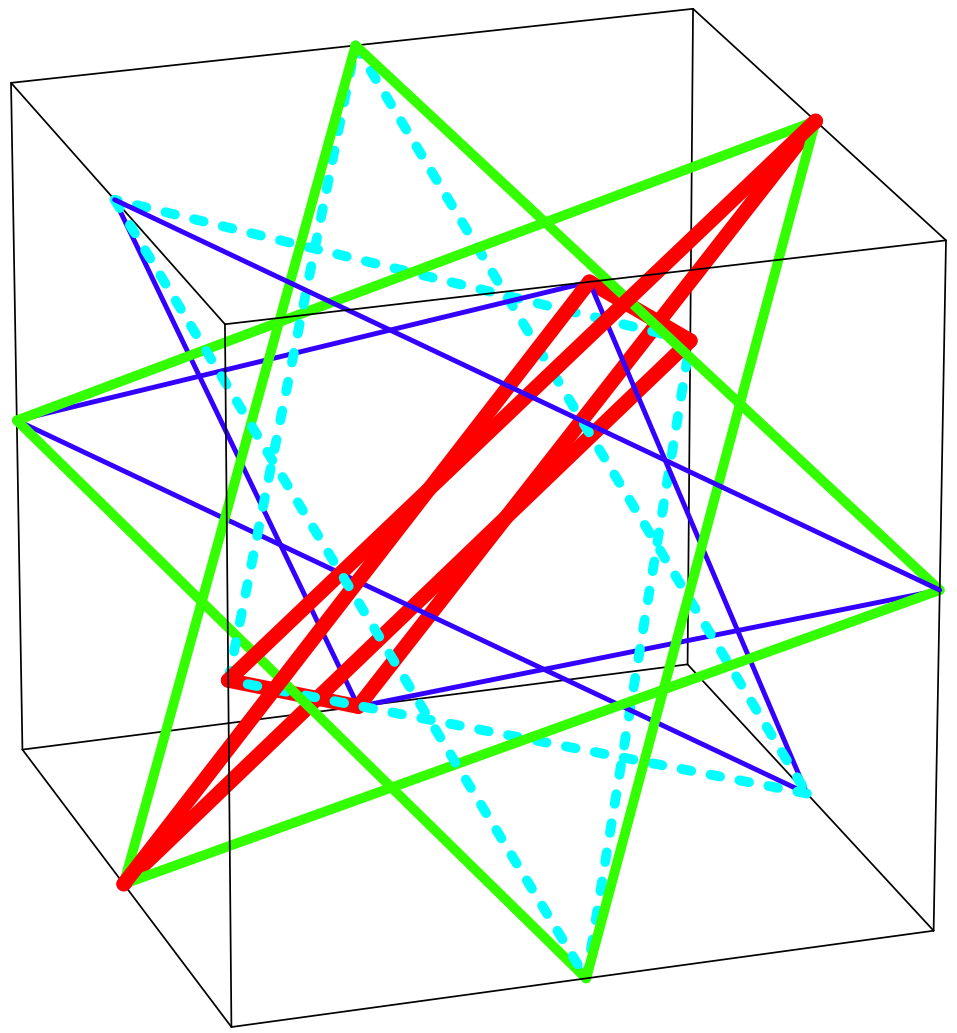

Figure 8. A ground state of the cuboctahedron formed by 4 Stars of David. 